\title{
Curvature forms with singullarities and Non-integral characteristic classes
}

\author{
By Akira Asada \\ Department of Mathematics, Shinshu University \\ (Received, Oct. 10, 1985)
}

\section{Introduction}

This is the detailed exposition of [7]. The outline of the paper is as follows: In $\S 1$, we define the 2-dimensional non-abelian de Rham set $H^{1}\left(M, \mathscr{H}^{1}\right)$. Here $M$ is a smooth manifold and $\mathscr{H}^{1}$ is the sheaf of germs of smooth matrix valued 1forms $\theta$ such that $d \theta+\theta_{\wedge} \theta=0$. A bijection between $\mathrm{H}^{1}\left(M, \mathscr{M}^{1}\right)$ and a set of special classes of matrix valued 2 -forms on $M$ are also defined. In a sense, these, 2 -forms can be regarded as curvature forms with singularities (singular gauge fields) (cf. [11], [17], [23], [24], [25]). Since we have obtained 2-dimensional non-abelian Poincaré lemma (local integration theorem of the eguation $d \theta+\theta_{\wedge} \theta=\Theta,[10]$ ), the results of this section will be improved in future. In $\$ 2$, we define the cohomology sets $\mathrm{H}^{2}\left(M, \mathrm{G}_{t}\right)$ and $\mathrm{H}^{2}\left(M, \mathrm{G}_{d}\right), \mathrm{G}=\mathrm{GL}(n, \mathrm{C})$. Here $\mathrm{G}_{t}$ and $\mathrm{G}_{d}$ are sheaves of germs of constant and smmooth $\mathrm{G}$-valued functions on $M$, respectively. Then we show the exactness of the following sequence

$$
\begin{aligned}
& 0 \stackrel{i}{\longrightarrow} \mathrm{H}^{0}\left(M, \mathrm{G}_{t}\right) \stackrel{\rho}{\longrightarrow} \mathrm{H}^{0}\left(M, \mathrm{G}_{d}\right) \stackrel{\delta}{\longrightarrow} \mathrm{H}^{0}\left(M, \mathscr{M}^{1}\right) \stackrel{i^{*}}{\longrightarrow} \mathrm{H}^{1}\left(M, \mathrm{G}_{t}\right) \longrightarrow \\
& \longrightarrow \mathrm{H}^{1}\left(M, \mathrm{G}_{d}\right) \stackrel{d^{*}}{\longrightarrow} \mathrm{H}^{1}\left(M, \mathscr{M}^{1}\right) \stackrel{\delta}{\longrightarrow} \mathrm{H}^{2}\left(M, \mathrm{G}_{t}\right) \stackrel{i^{*}}{\longrightarrow} \mathrm{H}^{2}\left(\mathrm{M}, \mathrm{G}_{d}\right) .
\end{aligned}
$$

This sequence is derived form the exact sequence of sheaves

$$
0 \longrightarrow \mathrm{G}_{t} \stackrel{i}{\longrightarrow} \mathrm{G}_{d} \stackrel{\rho}{\longrightarrow} \mathscr{H}^{1} \longrightarrow 0,
$$

and the exactness of the first 6-terms of the above sequence are known and used by several authors $([3],[15],[21],[22],[26])$. But the definitions and exactness of last 3-terms seem to be new. We note that our definition of $\mathrm{H}^{2}\left(M, \mathrm{G}_{t}\right)$ is different from former definitions ([13], [16], [19]). In fact, in our definition, $H^{2}$ $\left(M, \mathrm{G}_{t}\right)$ is not absolutely determined by the sheaf $\mathrm{G}_{t}$. Its definition depends on the above sheaf exact sequence. This is the reason why we have the cohomology exact sequence (cf. [14]). In $\$ 3$, we define the characteristic classes of the elements of $\mathrm{H}^{1}\left(M, \mathscr{M}^{1}\right)$. These are non-integral generalisation of the Chern classes (cf. [11], [23], [24], [25]). The characteristic classes of the elements of $\mathrm{H}^{2}\left(M, \mathrm{G}_{t}\right)$ and $\mathrm{H}^{2}$ 
$\left(M, \mathrm{G}_{d}\right)$ are also considered. Although there still remain many problems, we had some progress for the definition of the characteristic classes of the elements of $\mathrm{H}^{2}$ $\left(M, \mathrm{G}_{t}\right)$ and $\mathrm{H}^{2}\left(M, \mathrm{G}_{d}\right)([9])$. The results of $\S \S 1-3$ are constructed by the differential operator $\mathrm{d}$ and the group $\mathrm{G}=\mathrm{GL}(n, \mathbb{C})$ and $\mathrm{G}$ can be replaced by $\mathrm{U}(n)$ or $\mathrm{SU}(n)$. In $\S 4$, we give analogous theory replacing $\mathrm{d}$ by $\mathrm{D}$, an arbitrary differential operator over $M$. It is a refinement of the appendix of $[6]$ (cf. [1], [2], [5], [8]).

\section{§1. Non-abelian de $\mathbb{R h a m}$ theory of dimension 2}

1. Let $M$ be a connected paracompact smooth manifold. On $M$, we consider the following sheaves.

$\mathrm{G}_{t}$ : the sheaf of germs of constant $G$-valued functions.

$\mathrm{G}_{d}$ : the sheaf of germs of smooth $G$-valued functions.

$\mathrm{g}^{1}$ : the sheaf of germs of complex $(n, n)$-matrix valued 1 -forms.

$\mathscr{M}^{1}$ : the subsheaf of $g^{1}$ consisting of forms $\theta$ such that $d \theta+\theta_{\wedge} \theta=0$.

$\mathscr{A}^{2}$ : the image sheaf of $\hat{g}^{1}$ by the map $d^{e}$ defined by $d^{e} \varphi=d \varphi+\varphi \wedge \varphi$.

Using 2-dimensional non-abelian Poincaré lemma, we can give an intrinsic definition of $\mathscr{H}^{2}$. But in this paper, we use thisold definition.

Note 1 . If $G$ is a Lie group with the Lie algebra 8 such that the exponential map is onto. Then we can define the similar sheaves for G-valued functions and $g$-valued forms.

Note 2. We denote $\mathrm{G}_{\omega}, \mathscr{M}^{1}{ }_{\omega}$, etc., the corresponding sheaves for holomorphic maps. The similar notations are used for other categories.

Defilnition 1. We define the differential operators $\rho$ and $d^{e}$ respectively by

$$
\rho(g)=g^{-1} d g, \quad d^{e} \varphi=d \varphi+\varphi \wedge \varphi .
$$

The induced maps of $\rho$ and $d^{e}$ on the sheaves $\mathrm{G}_{d}$ and $\mathfrak{g}^{1}$ are also denoted by $\rho$ and $d^{e}$ respectively.

Note. $\rho$ is defined for Lie group valued functions, while $d^{e}$ is defined for Lie algebra valued forms. This is the reason of the inhomogenity of notations. For Lie algebra valued functions, $\mathrm{d}^{e}$ is defined by $d^{e} f=\rho\left(e^{f}\right)=d f+\sum_{n=1}^{\infty}\left((a d f)^{n} d f\right) /(n+1)$ ! ([8], [9]).

By definitions, we have the following exact sequence of sheaves.

$$
\begin{aligned}
& 0 \longrightarrow \mathrm{G}_{t} \stackrel{i}{\longrightarrow} \mathrm{G}_{d} \stackrel{\rho}{\longrightarrow} \mathscr{M}^{1} \longrightarrow 0, \\
& 0 \longrightarrow \mathscr{K}^{1} \stackrel{i}{\longrightarrow} \mathrm{g}^{1} \stackrel{d^{\mathrm{e}}}{\longrightarrow} \mathscr{K}_{2} \longrightarrow 0 .
\end{aligned}
$$

The 0-dimensional cohomology sets for these sheaves and 1-dimensional cohomology sets for $\mathrm{G}_{t}$ and $\mathrm{G}_{d}$ are known. But we need other 0-dimensional cohom- 
ology sets for $\mathscr{H}^{1}, g^{1}$ and $\mathscr{H}^{2}$ based on the action of gauge transformations. For these purposes, we take a locally finite open covering $\mathfrak{l}=\left\{U_{i}\right\}$ of $M$. We write $c_{i}$, $c_{i j}$, etc., the sections on $U_{i}, U_{i \cap} U_{j}$, etc.,

Definition 2. Let $h=\left\{h_{i}\right\}$ be an element of $\mathrm{C}^{0}\left(\mathfrak{u} . \mathrm{G}_{d l}\right)$. Then we define the actions of $h$ on $\mathrm{C}^{0}\left(\mathfrak{u}, g^{1}\right)$ and on $\mathrm{C}^{0}\left(\mathfrak{H}, \mathscr{M}^{2}\right)$ by

$$
\begin{aligned}
& h(\theta)=h_{i}\left(\theta_{i}-h_{i}^{-1} d h_{i}\right) h_{i}^{-1}, \quad \theta=\left\{\theta_{i}\right\} \in \mathrm{C}^{0}\left(\mathfrak{U}, \quad g^{1}\right), \\
& h(\Theta)=h_{i}\left(\Theta_{i}\right) h_{i}{ }^{-1}, \quad \Theta=\{\Theta\}_{i} \in \mathrm{C}^{0}\left(\mathfrak{u}, \mathscr{H}^{2}\right) .
\end{aligned}
$$

By definitions, we have

Lemma 1. For the above actions, the followings hold

$$
\begin{aligned}
& h_{1}\left(h_{2}(\theta)\right)=\left(h_{1} h_{2}\right)\langle\theta\rangle, h_{1}\left(h_{2}(\Theta)\right)=\left(h_{1} h_{2}\right)(\Theta), \\
& \mathrm{e}(\theta)=\theta, \mathrm{e}(\Theta)=\Theta, \mathrm{e}=\mathrm{e}_{i}(x), \text { the identity valued function. } \\
& d^{e}(h(\theta))=h\left(d^{e} \theta\right) . \\
& h\left(\mathrm{C}^{0}\left(\mathfrak{H}, \mathscr{M}^{1}\right)\right)=\mathrm{C}^{0}\left(\mathfrak{u}, \mathscr{M}^{1}\right) .
\end{aligned}
$$

2. $h=\left\{h_{i}\right\} \in \mathrm{C}^{0}\left(\mathfrak{u}, \mathrm{G}_{d}\right)$ also acts on $\left(\mathrm{C}^{1}\left(\mathfrak{u}, \mathrm{G}_{d}\right)\right.$ by the action

$$
h(\xi)=h_{i} g_{i j} h_{j}^{-1}, \xi=\left\{g_{i j}\right\} .
$$

By definition, we have

$$
h_{1}\left(h_{2}(\xi)\right)=\left(h_{1} h_{2}\right)(\xi), \mathrm{e}(\xi)=\xi .
$$

Definition 3. We set

$$
\mathrm{C}_{a}^{\mathfrak{1}}\left(\mathfrak{u}, \mathrm{G}_{d}\right)=\left\{\left\{g_{i j}\right\} \in \mathrm{C}^{1}\left(\mathfrak{u}, \mathrm{G}_{d}\right) \mid g_{i i}=\mathrm{e}, \quad g_{i j}=g_{j i}{ }^{-1}\right\} .
$$

By definition, we have

$$
h\left(\mathrm{C}^{1} a\left(\mathfrak{H}, \mathrm{G}_{d}\right)\right)=\mathrm{C}^{1} a\left(\mathfrak{U}, G_{d}\right) .
$$

Definition 4. Let $\xi=\left\{g_{i j}\right\}$ be an element of $\mathrm{C}^{1}\left(\mathfrak{u}, \mathrm{G}_{d}\right)$ and $\mathscr{C}$ be one of $\mathscr{H}^{1}, g^{1}$ or $\mathscr{H}^{2}$. Then we define the map $\delta \xi$ on $\mathrm{C}^{0}(\mathfrak{U}, \mathscr{C})$ by

(8)

$$
\delta_{\xi}(c)_{i j}=c_{j}-g_{j i} c_{i} g_{i j}, \quad c=\left\{c_{i}\right\} .
$$

By definition, if $\xi$ is in $\mathrm{C}_{a}^{1}\left(\mathfrak{h}, \mathrm{G}_{d}\right)$, we get

$$
\delta_{\xi}(c)_{i j}=c_{j}-g_{j i} c_{i} g_{i j}{ }^{-1}=c_{j}-g_{i j}\left(c_{i}\right) .
$$

Note. The image of $\delta_{\xi}$ is not in $\mathrm{C}^{1}(\mathfrak{H}, \mathscr{C})$ in general. For example, if $\xi \in \mathrm{C}_{a}^{1}$ (u, $\mathrm{G}_{d}$ ), we have

$$
d^{e}\left(\delta_{\xi}(\theta)\right)_{i j}=\delta_{\xi}\left(d^{e} \theta\right)_{i j}+\left[\rho\left(g_{i j}\right)-\delta_{\xi}(\theta)_{i j}, \quad g_{i j}^{-1} \theta_{i} g_{i j}\right]
$$

Here $[\varphi, \phi]$ means $\varphi_{\wedge} \phi-(-1)^{p q} \phi_{\wedge} \varphi, p=\operatorname{deg} \varphi, q=\operatorname{deg} \phi$.

Hereafter, we denote $t_{i j k}$ or $\delta g_{i j k}$ the map $g_{i j} g_{j k} g_{i k}{ }^{-1}$. 
Lemma 2. (i). If $\delta_{\xi}(c)=0$, then $\delta_{\xi} \rho(c)$ is epual to 0 if and only if

$$
h_{i j} c_{j} h_{j i}=c_{j}, \quad \xi=\left\{g_{i j}\right\} \text { and } \xi^{\prime}=\left\{h_{i j} g_{i j}\right\} .
$$

(ii). If $\xi \in \mathrm{C}^{1} a\left(\mathfrak{u}, \mathrm{G}_{d d}\right)$ and $\delta_{\xi}(c)$ is equal to 0 , then

$$
t_{i j k}{ }^{-1} c_{i} t_{i j k}=c_{i} \text {. }
$$

(iii). If $\Theta$ is in $\mathrm{C}\left(\mathfrak{H}, \mathscr{H}^{2}\right)$ and $\delta_{\xi} \Theta=0$, then $\delta_{h(\xi)}(h(\Theta))$ is equal to 0 . If $\theta$ is in $\mathrm{C}^{0}\left(\mathfrak{H}, g^{1}\right)$ and $\delta_{\xi}(\theta)=0$, then $\delta_{h(\xi)}(h(\theta))$ is equal to 0 if and only if

$$
\delta_{\xi}(\rho(h))=0 .
$$

(iv). If $\xi=\left\{g_{i j}\right\} \in \mathrm{C}^{1} a\left(\mathfrak{H}, \mathrm{G}_{d}\right)$ and $\delta_{\xi}(\theta)=0$, then $\delta_{\xi}\left(d^{e} \theta\right)$ is equal to 0 if and only if

$$
\left[\rho\left(g_{i j}\right), \theta_{j}\right]=0 .
$$

Proof. (i) and (ii) follow from the definitions. Since

$$
\delta_{h(\xi)}(h(\Theta))_{i j}=h_{j}\left(\delta_{\xi}(\Theta)_{i j}\right) h_{j}^{-1},
$$

we have the first assertion of (iii). The second assertion follows from $\delta_{h(\xi)}(h(\theta))_{i j}=$ $h_{j}\left(\delta_{\xi}(\theta)_{i j}-\delta_{\xi} \rho(h)_{i j}\right) h_{j}^{-1}$. Since $g_{i j^{-1}} \theta_{i} g_{i j}=\theta_{j}$ if $\delta_{\xi}(\theta)=0$, we have (iv) by (9).

Note. If $h$ satisfies (12) for $\xi \in \mathrm{C}_{a}^{1}\left(\mathfrak{U}, \mathrm{G}_{d}\right)$, then we have

$$
-\rho\left(h_{i}\right) \wedge \rho\left(h_{j}\right)=-\left[\rho\left(g_{i j}\right), g_{i j}^{-1} \rho\left(h_{i}\right) g_{i j}\right]-g_{i j^{-1}} \rho\left(h_{i}\right) g_{i j \wedge} g_{i j}^{-1} \rho\left(h_{i}\right) g_{i j}
$$

Hence we have

$$
\left[\rho\left(g_{i j}\right), \rho\left(h_{j}\right)\right]=0 .
$$

By this equality, if $\delta_{\xi}\left(d^{e} \theta\right)=\delta_{h(\xi)}(h(\theta))=0$, then we have $\delta_{h(\xi)}\left(d^{e}(h(\theta))=0\right.$.

Definition 5. We assume $\left.\xi=\left\{g_{i j}\right\} \in \mathrm{C}^{1} a(1), \mathrm{G}_{d}\right)$. Then we set

$$
\begin{gathered}
Z_{d}^{0}\left(\mathfrak{H}, \mathscr{M}^{1}\right)=\left\{\theta \in \mathrm{C}^{0}\left(\mathfrak{H}, \mathscr{H}^{1}\right) \mid \delta_{\xi} \theta=0 \text { for some } \xi \text { such that }\left[\rho\left(g_{i j}\right), \theta_{j}\right]=0\right\} . \\
Z_{d}^{0}\left(\mathfrak{H}, \theta^{1}\right)=\left\{\theta \in \mathrm{C}^{0}\left(\mathfrak{H}, g^{1}\right) \mid \delta_{\xi} \theta=0 \text { for some } \xi \text { such that }\left[\rho\left(g_{i j}\right), \theta_{j}\right]=0\right\} . \\
Z_{d}^{0}\left(\mathfrak{H}, \mathscr{M}^{2}\right)=\left\{\Theta \in \mathrm{C}^{0}\left(\mathfrak{H}, \mathscr{M}^{2}\right) \mid \delta_{\xi} \Theta=0 \text { for some } \xi \text { such that } \rho(\xi)=\delta_{\xi}(\theta),\right. \\
\left.\Theta=d^{e} \theta, \text { and } t_{i j k}{ }^{-1} \theta_{i} t_{i j k}=\theta_{i}\right\} .
\end{gathered}
$$

Definition 6. For $\theta \in Z^{0}{ }_{d}\left(\mathfrak{u}, \mathscr{H}^{1}\right)$ or $Z^{0}{ }_{d}\left(\mathfrak{u}, \theta^{1}\right)$, we call $h \in \mathrm{C}^{0}\left(\mathfrak{u}, \mathrm{G}_{d}\right)$ is an admissible action if

$$
\delta_{\xi}(\rho(h))=0, \quad \delta_{\xi} \theta=0 .
$$

Definition 7. For $\Theta \in Z_{d}^{0}\left(\mathfrak{H}, \mathscr{M}^{2}\right)$, we call $h$ is an admissible action if

$$
\delta_{\hat{\xi}} \Theta=0, \quad \rho\left(h_{i}\right)=t_{i j k}{ }^{-1} \rho\left(h_{i}\right) t_{i j k} .
$$

Since we have 


$$
\begin{aligned}
& \rho\left(h_{1} h_{2}\right)=\rho\left(h_{2}\right)+h_{2}^{-1} \rho\left(h_{1}\right) h_{2}, \\
& \delta_{\xi}\left(\rho\left(h_{1} h_{2}\right)\right\rangle_{i j}=\delta \xi \rho\left(h_{2}\right)_{i j}+h_{2, j^{-1}}\left(\delta_{h_{2}(\xi)} \rho\left(h_{2}\right)\right\rangle_{i j} h_{2, j},
\end{aligned}
$$

these actions are well defined.

Definition 8. The limit sets of $Z^{0}{ }_{d}\left(\mathfrak{U}, \mathscr{H}^{1}\right), Z^{0}{ }_{d}\left(\mathfrak{U}, \mathfrak{g}^{1}\right)$ and $Z_{d}^{0}\left(\mathfrak{H}, \mathscr{H}^{2}\right)$ with respect to the refinement of the covering $\mathfrak{U}$, are denoted by $Z_{d}^{0}\left(M, \mathscr{M}^{1}\right), Z_{d}^{0}\left(M, \mathbb{\theta}^{1}\right)$ and $\mathrm{Z}^{0}{ }_{d}\left(M, \mathscr{M}^{2}\right)$ respectively.

By definitions 6,7 , admissible actions are defined on $Z_{d}^{0}\left(M, \mathscr{M}^{1}\right), Z^{0}{ }_{d}\left(M, \theta^{1}\right)$ and $Z^{0}{ }_{d}\left(M, \mathscr{M}^{2}\right)$.

Definition 9. The quotient sets of $Z^{0}{ }_{d}\left(M, \mathscr{M}^{1}\right), Z^{0}{ }_{d}\left(M, g^{1}\right)$ and $Z^{0}{ }_{d}\left(M, \mathscr{M}^{2}\right)$ by the admissible actions are denoted $\mathrm{H}_{d}^{0}\left(M, \mathscr{M}^{1}\right), \mathrm{H}^{0} d\left(M, \mathfrak{g}^{1}\right)$ and $\mathrm{H}_{d}^{0}\left(M, \mathscr{M}^{2}\right)$. They called the 0-dimensional cohomolgy sets.

By the definitions and Lemma 2, we have the following exact sequence

$$
0 \longrightarrow Z^{0} d\left(M, \mathscr{M}^{1}\right) \stackrel{i}{\longrightarrow} Z_{d}^{0}\left(M, \mathrm{~g}^{1}\right) \stackrel{d^{\mathrm{e}}}{\longrightarrow} \mathrm{Z}^{0}{ }_{d}\left(M, \mathscr{M}^{2}\right) .
$$

By (5) and Lemma 2, $d^{e}$ also induces the map $d^{e}: \mathrm{H}^{0} d\left(M, \mathrm{~g}^{1}\right) \longrightarrow \mathrm{H}^{0} d\left(M, \mathscr{M}^{2}\right)$.

3. Definition 10. Let $\mathscr{C}$ be $\mathscr{H}^{1}$ or $\mathbb{G}^{1}$ an $\xi \in \mathrm{C}^{1}\left(\mathfrak{l}, \mathrm{G}_{d}\right)$. Then we define the map $\delta_{\xi}$ on $\mathrm{C}^{1}(\mathfrak{u}, \mathscr{C})$ by

$$
\delta_{\xi}(\omega)_{i j k}=\omega_{j k}-\omega_{i k}+g_{k j} \omega_{i j} g_{j k}, \quad \xi=\left\{g_{i j}\right\} .
$$

Note. In general, $\delta_{\xi}\left(\mathrm{C}^{1}\left(\mathfrak{H}, \mathscr{K}^{1}\right)\right)$ is not contained in $\mathrm{C}^{2}\left(\mathfrak{U}, \mathscr{K}^{1}\right)$.

By definition, $\delta_{\xi}\left\langle\delta_{\xi} \theta\right)$ is equal to 0 if and only if $g_{k i} \theta_{i} g_{i k}=g_{k j} g_{j i} \theta_{i} g_{i j} g_{j k}$ for any $i, j, k$. If $\xi \in \mathrm{C}_{a}^{1}\left(\mathfrak{u}, \mathrm{G}_{d}\right)$, then

$$
\delta_{\xi}(\omega)_{i j k}=\omega_{j k}-\omega_{i k}+g_{j k}^{-1} \omega_{i j} g_{j k} .
$$

Definition 11. Let $\xi$ be in $\mathrm{C}_{a}^{\mathfrak{1}}\left(\mathfrak{u}, \mathrm{G}_{d}\right)$. Then we set

$\mathrm{Z}^{1}\left(\mathfrak{H}, \mathscr{M}^{1}\right)=\left\{\omega \in \mathrm{C}^{1}\left(\mathfrak{u}, \mathscr{M}^{1}\right) \mid \omega=\rho\left(g_{i j}\right)\right.$ and $\delta_{\xi} \omega=0$ for some $\left.\xi=\left\{g_{i j}\right\}\right\}$,

$Z^{1}\left(\mathfrak{u}, a^{1}\right)=\left\{\omega \in \mathbb{C}^{1}\left(\mathfrak{u}, g^{1}\right) \mid \delta \xi \omega=0\right.$ for some $\left.\xi\right\}$.

Definition 12. The elements $\omega$ and $\omega^{\prime}$ of $Z^{1}\left(\mathfrak{H}, \mathscr{M}^{1}\right)$ are said to be cohomologous and denoted $\omega \sim \omega^{\prime}$ if there exists $h=h_{1} \in \mathrm{C}^{0}\left(\mathfrak{H}, \mathrm{G}_{d}\right)$ such that

$$
\begin{aligned}
& d\left(h_{i} t_{i j k} h_{i}^{-1}\right)=0 \text { for any } i, j, k, \omega_{i j}=\rho\left(g_{i j}\right), \\
& \xi=\left\{g_{i j}\right\} \in \mathrm{C}_{a}^{1}\left(\mathfrak{U}, \mathrm{G}_{d}\right) \text { and } \delta_{\xi}(\omega)=0 . \\
& \omega_{i j}{ }^{\prime}=h_{j}\left(\omega_{i j}-\delta \xi \rho(h)_{i j}\right) h_{j}^{-1} .
\end{aligned}
$$

Note. By $(16)$, we have $\omega_{i j}{ }^{\prime}=\rho\left(h_{i} g_{i j} h_{j}{ }^{-1}\right)$. This $\omega^{\prime}$ satisfies $\delta_{\xi^{\prime}}\left(\omega^{\prime}\right)=0$, where $\xi^{\prime}=\left\{h_{i} g_{i j} h_{j}^{-1}\right\} \in \mathrm{C}_{a}^{1}\left(\mathfrak{U}, \mathrm{G}_{d}\right)$.

Definition 13. The elements $\omega$ and $\omega^{\prime}$ of $Z^{1}\left(11, g^{1}\right)$ are said to be cohomologous and denoted $\omega \sim \omega^{\prime}$ if there exist $h=\left\{h_{i}\right\} \in \mathrm{C}^{\circ}\left(\mathfrak{u}, \mathrm{G}_{d}\right)$ and $\theta=\left\{\theta_{i}\right\} \in \mathrm{C}^{\circ}\left(\mathfrak{u}, \theta^{1}\right)$ such that 


$$
\omega_{i j}{ }^{\prime}=h_{j}\left(\omega_{i j}-\delta_{\xi}(\theta)^{2}{ }_{j}\right) h_{j}{ }^{-1} \text {. }
$$

Definition 14. $\mathrm{H}^{1}\left(\mathfrak{H}, \mathscr{K}^{1}\right)$ and $\mathrm{H}^{1}\left(\mathfrak{H}, g^{1}\right)$ are defined to be the quotient sets of $Z^{1}\left(\mathfrak{U}, \mathscr{H}^{1}\right)$ and $Z^{1}\left(\mathfrak{u}, g^{1}\right)$ by the cohomology relations.

Note. The sets of coboundaries are given by $B^{1}\left(\mathfrak{H}, \mathscr{A}^{1}\right\}=\left\{\omega \mid \omega=\delta_{\xi}(\theta), \quad \theta \in \mathrm{C}^{0}\right.$ $\left.\left(\mathfrak{u}, \mathscr{K}^{1}\right), \omega=\rho(\xi)\right\}$ and $\mathrm{B}^{1}\left(\mathfrak{H}, \hat{\theta}^{1}\right)=\left\{\omega \mid \omega=\delta_{\xi}(\theta), t_{i j k}{ }^{-1} \theta_{i} t_{i j k}=\theta_{i}\right\}$.

Note. If $\omega \in \mathrm{C}^{1}\left(\mathfrak{U}, \mathscr{M}^{1}\right)$ satisfies $\omega_{j k}-\omega_{i k}+g_{j k}{ }^{-1} \omega_{i j} g_{j k}=0, \omega_{i j}=\rho\left(g_{i j}\right)$ for some $\left\{g_{i j}\right\}$, then we have

(i) $g_{j k}{ }^{-1} \omega_{i j} g_{j k}$ does not depend on the choice of $\left\{g_{i j}\right\}$,

(ii) $\omega_{i i}=0$ and $\omega_{i j}=-g_{j i} \omega_{j i} g_{j i}{ }^{-1}$.

By (ii), we may assume $\omega=\rho(\xi), \xi \in \mathrm{C}^{1}{ }_{a}\left(\mathfrak{H}, \mathrm{G}_{d}\right)$.

Proposition 1. (i) Let $\xi=\left\{g_{i j}\right\}$ be in $\mathrm{C}_{a}^{1}$ ( $\left.\mathfrak{u}, \mathrm{G}_{d}\right)$. Then $\rho(\xi)$ belongs to $\mathrm{Z}^{1}\left(\mathfrak{u}, \mathscr{M}^{1}\right)$ if and only if $t_{i j k}=\left(\delta_{\xi}\right)_{i j k}$ is a constant map for any $i, j, k$.

(ii) $\mathrm{Z}^{1}\left(\mathfrak{H}, g^{1}\right)$ is equal to $\mathrm{B}^{1}\left(\mathfrak{H}, g^{1}\right)$.

Proof. Since we have

$$
t_{i j k}^{-1} d\left(t_{i j k}\right)=g_{i k}\left(g_{j k}^{-1} \omega_{i j} g_{j k}+\omega_{j k}-\omega_{i k}\right) g_{i k}{ }^{-1},
$$

$d\left(t_{i j k}\right)$ is equal to 0 if $\omega \in Z^{1}\left(\mathfrak{u}, \mathscr{K}^{1}\right)$. On the other hand, if $d\left(t_{i j k}\right)$ is equal to $0, \omega$ $=\rho(\xi)$ satisfies $\delta_{\xi} \omega=0$. Hence we have (i).

Since we have $g_{j k}{ }^{-1} g_{i j^{-1}} \omega_{k i} g_{i j} g_{j k}=g_{j k}{ }^{-1}\left(\omega_{k j}-\omega_{i j}\right) g_{j k}=-\omega_{j k}+\left(\omega_{i j}-\omega_{i k}\right)=-\omega_{i k}=$ $g_{i k^{-1}} \omega_{k i} g_{i k}$ if $\delta \xi \omega=0$, we get $t_{i j k^{-1}} \omega_{k i} t_{i j k}=\omega_{k i}$ if $\delta \xi \omega=0$. Hence to take a smooth partition of unity $\left\{e_{i}\right\}$ subordinate to $U$,

$$
\theta_{i}=\sum_{U_{k} \cap U_{i} \neq \neq \phi} e_{k} \omega_{k i}
$$

satisfies $(15)^{\prime}$. Then, since

$$
\theta_{j}-g_{i j^{-1}} \theta_{i} g_{i j}=\sum_{U_{k} \cap U_{i} \cap U_{j \neq \phi}} e_{k}\left(\omega_{k j}-g_{i j}{ }^{-1} \omega_{k i} g_{i j}\right)=\omega_{i j}
$$

$\omega$ is equal to $\delta_{\xi}(\theta)$. Therefore we obtain (ii).

Corollary 1. For elements of $\left.Z^{1} \mathfrak{u}, \mathscr{M}^{1}\right),(15)^{\prime}$ follows from (15).

Proof. Since $t_{i j k}$ is a constant, we get $h_{i}^{-1}\left(d\left(h_{i} t_{i j k} h_{i}^{-1}\right)\right) h_{i}=\rho\left(h_{i}\right) t_{i j k}-t_{i j k} \rho\left(h_{i}\right)=$ 0 . Hence wehave Corollary.

Corollary 2. $\mathrm{H}^{1}\left(\mathfrak{u}, \mathfrak{g}^{1}\right)$ is equal to $\{0\}$.

Note. Corollary 2 does not hold in another category. For example, $\mathrm{H}^{1}\left(\mathfrak{u}, g^{1} \omega\right)$ may not be equal to $\{0\}$ (cf. [6], [7], [22]).

Definition 15. We set $\mathrm{C}_{c}\left(\mathfrak{H}, \mathrm{G}_{d}\right)=\left\{\left\{g_{i j}\right\} \in \mathrm{C}^{1}{ }_{a}\left(\mathfrak{H}, \mathrm{G}_{d}\right) \mid \delta g_{i j k}\right.$ is a constant for any $i, j, k\}$.

Let $\mathfrak{B}=\left\{V_{j} \mid j \in \mathbb{J}\right\}$ be a refinement of $\mathfrak{U}=\left\{U_{i} \mid i \in \mathbb{I}\right\}$ and $\tau, \tau^{\prime}: \mathbb{J}>\mathbb{I}$ the maps 
such that $V_{j} \subset U_{\tau(i) \cap} U_{\tau^{\prime}(i)}$. Then $\left\{h_{i}\right\}=\left\{g_{\tau(i) \tau^{\prime}(i)}\right\}$ gives a chain homotopy between $\tau^{*}$ and $\tau^{*}$.

Definition 16. We set $\mathrm{H}^{1}\left(M, \mathscr{M}^{1}\right)$ the limit set $\lim \left\{\mathrm{H}^{1}\left(\mathfrak{U}, \mathscr{M}^{1}\right) \mid \tau^{*}\right\}$.

Note. $\mathrm{H}^{1}\left(M, g^{1}\right)$ is defined ay the same way. But by Corollary 2 of proposition 1 , we have

$$
\mathrm{H}^{1}\left(M, \mathrm{~B}^{1}\right)=\{0\} .
$$

4. Definition 17. Let $\bar{\Theta}$ and $\bar{\Theta}^{\prime}$ be elements of $\mathrm{H}^{0} d\left(M, \mathscr{H}^{2}\right)$. $\bar{\Theta}$ and $\bar{\Theta}^{\prime}$ are cohomologous if they have representatives $\Theta=\left\{\Theta_{i}\right\}$ and $\Theta^{\prime}=\left\{\Theta_{i}{ }^{\prime}\right\}$ such that $\delta_{\xi} \Theta=0$, $\delta_{\xi}$, $\Theta^{\prime}=0, \quad \Theta_{i}=d^{e} \theta_{i}, \quad \Theta_{i}{ }^{\prime}=d^{e} \theta_{i}{ }^{\prime}, \rho(\xi)=\delta \xi \theta, \rho\left(\xi^{\prime}\right)=\delta \xi, \quad \theta^{\prime}, \quad \xi=\left\{g_{i j}\right\}$ and $\xi^{\prime}=\left\{c_{i j} g_{i j}\right\}$, where $c_{i j}$ is a constant map.

Note. $\rho(\xi)=\delta_{\xi} \theta$ if and only if $\theta_{j}-g_{i j^{-1}}\left(\theta_{i}\right)=0$.

Definition 18. $\mathrm{H}^{2}{ }_{d R}(M, g)$ is defined as the quotient set of $\mathrm{H}^{0} d\left(M, \mathscr{H}^{2}\right)$ by the cohomology relation.

Theorem 1. There is a bijection between $\mathrm{H}_{d R}^{2}(M, 9)$ and $\mathrm{H}^{1}\left(M, \mathscr{M}^{1}\right)$.

Proof. We denote $\langle\Theta\rangle$ theclass of $\theta \in Z^{0}{ }_{d}\left(\mathfrak{u}, \mathscr{M}^{2}\right)$ in $\mathrm{H}^{2}{ }_{d R}(M, g)$. Then we $\delta_{\xi} \Theta=0, \quad \rho(\xi)=\delta_{\xi} \theta, \quad \xi \in \mathrm{C}^{1}{ }_{a}\left(\mathfrak{u}, \mathrm{G}_{d}\right)$ and $\theta \in \mathrm{C}^{0}\left(\mathfrak{U}, \theta^{1}\right)$. Then, since $t_{i j k^{-1}} \theta_{i} t_{i j k}=\theta_{i}$, we obtain

$$
\delta_{\xi}(\rho(\xi))=\delta_{\xi}\left(\delta_{\xi} \theta\right)=0 .
$$

Hence $\rho(\xi)$ belongs to $Z^{1}\left(\mathfrak{U}, M^{1}\right)$. If $\Theta^{\prime}$ is another representative of $\langle\Theta\rangle$ such that $\delta \xi, \Theta^{\prime}=0$, set $\xi=\left\{g_{i j}\right\}$ and $\xi^{\prime}=\left\{g_{i j}{ }^{\prime}\right\}$, we have

$$
g_{i j}^{\prime}=h_{i} c_{i j} g_{i j} h_{j}^{-1}
$$

where $c_{i j}$ is a constant and $\left\{h_{i}\right\}$ is admissible. Then, since $\rho\left(c_{i j} g_{i j}\right)=\rho\left(g_{i j}\right)$, we may assume $g_{i j}{ }^{\prime}=h_{i} g_{i j} h_{j}^{-1}$. Hence we have $\rho\left(\xi^{\prime}\right)_{i j}=h_{j}\left(\rho(\xi)_{i j}-\delta_{\xi} \rho(h)_{i j}\right) h_{j}^{-1}$ and $d\left(h_{i} t_{i j h} h_{i}{ }^{-1}\right)$ $=0$. Therefore $\rho(\xi) \sim \rho\left(\xi^{\prime}\right)$ and we can define the map $\iota: \mathrm{H}^{2} d R(M, g) \rightarrow \mathrm{H}^{1}\left(M, \mathscr{M}^{1}\right)$ by

$$
\iota(\langle\Theta\rangle)=\langle\rho(\xi)\rangle \text { : the class of } \rho(\xi) \text { in } \mathrm{H}^{1}\left(M, \mathscr{M}^{1}\right) .
$$

Since $\theta_{j}=\rho\left(g_{i j}\right)+g_{i j^{-1}} \theta_{i} g_{i j}$ if $\rho(\xi)=\delta_{\xi}(\theta)$, we have $\delta_{\xi} d^{e} \theta=0$ if $\rho(\xi)=\delta_{\xi}(\theta)$. Hence we have

$$
d \theta_{j}=-\rho\left(g_{i j}\right) \wedge \rho\left(g_{i j}\right)-\rho\left(g_{i j}\right), \quad g_{i j}{ }^{-1} \theta_{i} g_{i j}+g_{i j}{ }^{-1} d \theta_{i} g_{i j}
$$

Therefore $d^{e} \theta_{j}=g_{i j^{-1}} d^{e} \theta_{i} g_{i j}$, that is $\delta_{\xi} d^{e} \theta=0$. Let $\langle\omega\rangle$ be an element of $\mathrm{H}^{1}\left(M, \mathscr{M}^{1}\right)$. Then we set $\omega=\rho(\xi), \xi \in \mathrm{C}_{c}\left(\mathfrak{H}, \mathrm{G}_{d}\right)$. By Proposition 1, (ii), we can set $\rho(\xi)=\delta_{\xi}(\theta)$, $\theta \in \mathrm{C}^{0}\left(\mathfrak{H}, \mathfrak{g}^{1}\right)$. Then by the above calculation, $d^{e} \theta$ belongs to $Z_{d}^{0}\left(\mathfrak{H}, \mathscr{M}^{2}\right)$. If $\omega \sim \omega^{\prime}$, we set $\omega^{\prime}=\rho(h(\xi))$. Then $h \in \mathrm{C}^{0}\left(\mathfrak{U}, \mathrm{G}_{d}\right)$ defines an admissible action of $d^{e} \theta$. Hence we can define the map $\kappa: \mathrm{H}^{1}\left(M, \mathscr{M}^{1}\right) \rightarrow \mathrm{H}^{2}{ }_{d R}(M$, g) by

$$
\kappa(\langle\omega\rangle)=\left\langle d^{e} \theta\right\rangle, \omega=\rho(\xi)=\delta_{\xi} \theta .
$$


By the definitions of $\iota$ and $\kappa, \iota \kappa$ is the identity map of $\mathrm{H}_{d R}^{2}(M, g)$ and $\kappa \iota$ is the identity map of $\mathrm{H}^{1}\left(M, \mathscr{A}^{1}\right)$. Hence we have Theorem.

Note 1. The kernel of the surjection $\delta: \mathrm{H}^{0}{ }_{d}\left(M, \mathscr{M}^{2}\right) \rightarrow \mathrm{H}^{1}\left(M, \mathscr{M}^{1}\right)$ is given by $d^{e}\left(\mathrm{H}_{t}^{0}\left(M, g^{1}\right)\right), \mathrm{H}^{0}{ }_{t}\left(M, \theta^{1}\right)=\left\{\langle\theta\rangle \in \mathrm{H}^{0}{ }_{d}\left(M, g^{1}\right) \mid \delta_{\xi} \theta=0\right.$ for some $\left.\xi \in \mathrm{C}^{1}{ }_{a}\left(\mathfrak{l}, \mathrm{G}_{t}\right)\right\}$. Here $\mathrm{C}_{a}^{1}\left(\mathfrak{u}, \mathrm{G}_{t}\right)$ means $\mathrm{C}^{1}\left(\mathfrak{H}, \mathrm{G}_{t}\right)_{\cap} \mathrm{C}_{a}^{\mathfrak{1}}\left(\mathfrak{U}, \mathrm{G}_{d}\right)$.

Note 2. In holomorphic category, $\mathrm{H}^{1}\left(M, g^{1} \omega\right)$ may not be epual to $\{0\}$ and we have the following exact sequence

$$
0 \longrightarrow \mathrm{H}^{0}{ }_{t}\left(M, \mathscr{M}^{1}{ }_{\omega}\right) \stackrel{i^{*}}{\longrightarrow} \mathrm{H}^{0}{ }_{t}\left(M, \mathrm{\theta}^{1} \omega\right) \stackrel{d^{\mathrm{e}}}{\longrightarrow} \mathrm{H}^{0}{ }_{d}\left(M, \mathscr{H}^{2}{ } \omega\right) \longrightarrow \mathrm{H}^{1}\left(M, \mathscr{H}^{1}{ }_{\omega}\right) \stackrel{i^{*}}{\longrightarrow} \mathrm{H}^{1}\left(M, \mathrm{~g}^{1} \omega\right)
$$

The image $i^{*}(\langle\omega\rangle)$ of $\langle\omega\rangle \in \mathrm{H}^{1}\left(M, \mathscr{M}^{1}{ }^{1}\right)$ is the obstruction class of $\langle\omega\rangle$ to be in $\delta$ image. Especially, if $\omega=\rho^{*}(\xi), \xi \in \mathrm{H}^{1}\left(M, \mathrm{G}_{\omega}\right), i^{*} \rho^{*}(\xi)$ is the obstruction class of $\xi$ to have a holomorphic connection.

\section{§2. The cohomology sets $\mathrm{H}^{2}\left(M, \mathrm{G}^{t}\right)$ and $\mathrm{H}^{2}\left(M, \mathrm{G}_{\boldsymbol{d}}\right)$}

5. Definition 19. Let $\xi=\left\{g_{j j}\right\}$ be in $\mathrm{C}_{a}^{1}\left(\mathfrak{H}, \mathrm{G}_{d}\right)$. Then we define a map $\delta_{\xi}$ on $\mathrm{C}^{2}\left(\mathfrak{H}, \mathrm{G}_{t}\right)\left(\right.$ resp. on $\left.\mathrm{C}^{2}\left(\mathfrak{H}, \mathrm{G}_{d}\right)\right)$ by

$$
\left(\delta_{\xi} c\right)_{i_{0} i_{1} i_{2} i_{3}}=g_{i_{0} i_{1}} c_{i_{1} i_{2} i_{3}} g_{i_{0 i} i_{1}}{ }^{-1} c_{i_{0} i_{1} i_{3}} c_{i_{0} i_{2} i_{3}}{ }^{-1} c_{i_{0 i} i_{2}{ }^{-1}} .
$$

By definition, we have

Lemma 3. (i) $\delta_{\xi}$ maps $\mathrm{C}^{2}\left(\mathfrak{H}, \mathrm{G}_{d}\right)$ into $\mathrm{C}^{\mathrm{3}}\left(\mathfrak{u}, G_{d}\right)$.

(ii) $\delta_{\xi} c=\mathrm{e}$ if and only if

$$
c_{i_{0} i_{1 i} i_{2}} c_{i_{0} i_{2} i_{3}}=g_{i_{0} i_{1}} c_{i_{1} i_{2} i_{3}} g_{i_{0} i_{1}}^{-1} c_{i_{0} i_{1} i_{3}} \text {. }
$$

(ii) If $c \in \mathrm{C}^{2}\left(\mathfrak{H}, \mathrm{G}_{t}\right)$ satisfies $\delta_{\xi} c=\mathrm{e}, g_{i_{0} i_{1}} c_{i_{1} i_{2} i_{3}} g_{i_{0} i_{1}}^{-1}$ is a constant map.

(iii) If $\delta \xi c=\delta \xi, c=\mathrm{e}$, we get $h_{i_{0} i_{1}} c_{i_{1} i_{2} i_{3}} h_{i_{0} i_{1}}{ }^{-1}=c_{i_{1} i_{2} i_{3}}$, where $\xi=\left\{g_{i j}\right\}$ and $\xi^{\prime}=$ $\left\{h_{i j} g_{i j}\right\}$.

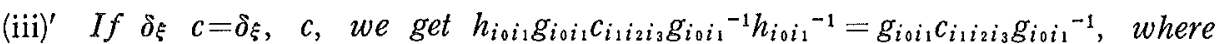
$\xi=\left\{g_{i j}\right\}$ and $\xi^{\prime}=\left\{h_{i j} g_{i j}\right\}$.

(iv) If $c=\delta_{\xi}$, that is, $c_{i_{0} i_{1} i_{2}}=g_{i_{0} i_{1}} g_{i_{1} i_{2}} g_{i_{0} i_{2}}{ }^{-1}$, then $\delta_{\xi}(\delta \xi)=\mathrm{e}$.

(v) Define the action of $h=\left\{h_{i}\right\} \in \mathrm{C}^{0}\left(\mathfrak{u}, G_{d}\right)$ on $\mathrm{C}^{2}\left(\mathfrak{u}, G_{t}\right)\left(\right.$ on $\left.\mathrm{C}^{2}\left(\mathfrak{u}, G_{d}\right)\right)$ by $h(c)=$ $h_{i_{0}} c_{i_{0} i_{1} i_{2}} h_{i_{0}}{ }^{-1}$, we get

$$
\delta_{h(\xi)}(h(c))=h\left(\delta_{\xi}(c)\right), \quad h(a)=h_{i_{0}} a_{i_{0 i 1} i_{2 i} g_{i 0}} h_{i_{0}}{ }^{-1}
$$

Example. If $\delta_{\xi} c=\mathrm{e}$ and $\xi=h_{i} h_{j}^{-1}$, then $c^{\prime}=h^{-1}(c)$ satisfies

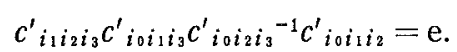

Definition 20. Let $c$ be an element of $\mathrm{C}^{2}\left(\mathfrak{H}, \mathrm{G}_{t}\right)$. Then $h \in \mathrm{C}^{0}\left(\mathfrak{H}, \mathrm{G}_{d}\right)$ is called $c$-admissible if $h(c)$ belongs to $\mathrm{C}^{2}\left(\mathfrak{H}, \mathrm{G}_{t}\right)$.

Lemma 4. Let $\delta_{\xi} c=\mathrm{e}, \xi=\left\{g_{i j}\right\}$ and assume $\xi^{\prime}=\left\{a_{i j} g_{i j}\right\}$ is in $\mathrm{C}_{a}{ }_{a}\left(\mathfrak{H}, \mathrm{G}_{d}\right)$. Then 
to set

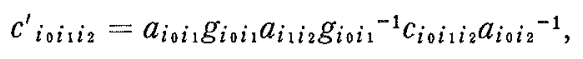

$\xi^{\prime} c^{\prime}=\mathrm{e}$ if and only if $a=\left\{a_{i j}\right\}$ satisfies

$$
\left.\left(g_{i_{0} i_{1}}{ }^{-1} \delta c_{i_{0} i_{12}}\right)\left(g_{i_{0} i_{2}} a_{i_{2} i_{3}} g_{i_{0} i_{2}}{ }^{-1}\right)=\left(g_{i_{1} i_{2}} a_{i_{2} i_{3}} g_{i_{11} i_{2}}{ }^{-1}\right) g_{i_{0} i_{1}}{ }^{-} c_{i_{0} i_{1} i_{2}}\right) .
$$

Proof. By (18), $\delta_{\xi^{\prime}} c^{\prime}=\mathrm{e}$ if and only if

$$
c^{\prime}{ }_{i_{0} i_{1} i_{2}} c^{\prime}{ }_{i_{0} i_{2} i_{3}}=a_{i_{0} i_{1}} g_{i_{0} i_{1}} c^{\prime}{ }_{i_{1} i_{2} i_{3}} g_{i_{0} i_{1}}{ }^{-1} a_{i_{0} i_{1}}{ }^{-1} c^{\prime}{ }_{i_{0} i_{1} i_{3}} .
$$

By (19), we get

$$
\begin{aligned}
& c^{\prime}{ }_{i_{0} i_{1} i_{2}} c^{\prime}{ }_{i_{0} i_{2} i_{3}}=a_{i_{0} i_{1}} g_{i_{0} i_{1}} a_{i_{1} i_{2}} g_{i_{0} i_{1}}{ }^{-1} c_{i_{0} i_{1} i_{2}} a_{i_{0} i_{2}}{ }^{-1} a_{i_{0} i_{2}} g_{i_{0} i_{2}} a_{i_{2} i_{3}} g_{i_{0} i_{2}}{ }^{-1} c_{i_{0} i_{2} i_{3}} a_{i_{0} i_{3}}{ }^{-1} \text {, }
\end{aligned}
$$

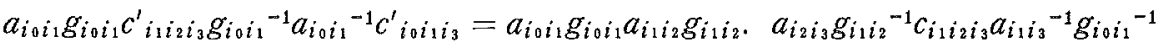

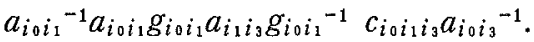

Hence $(18)^{\prime}$ follows from

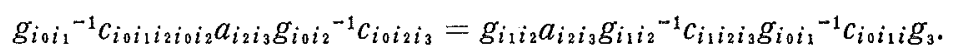

But since $g_{i_{0}}{ }^{-1} c_{i_{0} i_{1} i_{3}}=c_{i_{1} i_{2} i_{3}}{ }^{-1} g_{i_{0} i_{1}}{ }^{-1} c_{i_{0} i_{1} i_{2}} c_{i_{0} i_{2} i_{3}}$ by (18), (18) holds if and only if the equality (20) holds. Hence we have Lemma.

Note. If $c=\delta_{\xi},(20)$ always holds. In fact, we get $\left(g_{i_{0 i 1}}^{-1} c_{i_{0 i} i_{2}}\right)\left(g_{i_{0 i} i_{2}} a_{i_{2 i} i_{3}} g_{i_{0} i_{2}}{ }^{-1}\right)$ $=\left(g_{i_{1} i_{2}} a_{i_{2} i_{3}} g_{i_{1} i_{2}}^{-1}\right)\left(g_{i_{0 i} i_{1}}^{-1} c_{i_{0} i_{1} i_{2}}\right)=g_{i_{1 i_{2}}} a_{i_{2} i_{3}} g_{i_{0} i_{2}}{ }^{-1}$.

6. Definition 21. We set

$$
\begin{aligned}
& Z^{2}\left(\mathfrak{H}, \mathrm{G}_{t}\right)=\left\{c \in \mathrm{C}^{2}\left(\mathfrak{U}, \mathrm{G}_{t}\right) \mid \delta_{\xi} c=\mathrm{e} \text { for some } \xi \in \mathrm{C}^{1}{ }_{c}\left(\mathfrak{U}, \mathrm{G}_{d}\right)\right\} . \\
& \mathrm{Z}^{2}\left(\mathfrak{u}, \mathrm{G}_{d}\right)=\left\{c \in \mathrm{C}^{2}\left(\mathfrak{H}, \mathrm{G}_{d}\right) \mid \delta_{\xi} c=\mathrm{e} \text { for some } \xi \in \mathrm{C}^{1}{ }_{c}\left(\mathfrak{U}, \mathrm{G}_{d}\right)\right\} .
\end{aligned}
$$

Note 1 . The definitions of $Z^{2}\left(\mathfrak{H}, \mathrm{G}_{t}\right)$ and $Z^{2}\left(\mathfrak{u}, \mathrm{G}_{d}\right)$ depend on the domain of $\xi$. From this point of view, the notations $Z^{2}\left(\mathfrak{H}, G_{t}\right)_{G_{d}}$ and $Z^{2}\left(\mathfrak{H}, G_{d}\right)_{\mathfrak{G} a}$ are more exact. But we do not use these notations.

Note 2. In [8], we only assume the domain of $\xi$ to be $\mathrm{C}_{a}{ }_{a}\left(\mathfrak{H}, \mathrm{G}_{d}\right)$. But the research on 3-dimensional theory suggests above definitions are more convenient.

Definition 22. $c, c^{\prime} \in Z^{2}\left(\mathfrak{H}, \mathrm{G}_{t}\right)$ (resp. $Z^{2}\left(\mathfrak{H}, \mathrm{G}_{d}\right)$ ) are said to be cohomologous and denoted in symbols $c \sim c^{\prime}$ if there exists $a \in \mathrm{C}^{1}\left(\mathfrak{H}, \mathrm{G}_{t}\right)$ (resp. $\mathrm{C}^{1}\left(\mathfrak{H}, \mathrm{G}_{d}\right)$ ) such that $\xi^{\prime}=\left\{a_{i j} g_{i j}\right\} \in \mathrm{C}_{c}^{1}\left(\mathfrak{H}, \mathrm{G}_{d}\right)$ satisfies (20) for $c$ and $c^{\prime}$ is expressed by (19). Here $\delta_{\xi} c=\mathrm{e}$ and $\xi=\left\{g_{i j}\right\}$.

Lemma 5. (i) $c \sim c^{\prime}$ is an equivalence relation.

(ii) If $c \sim c^{\prime}$ and $h(a) \in \mathrm{C}^{1}\left(\mathfrak{H}, \mathrm{G}_{t}\right)$, then $h(c) \sim h\left(c^{\prime}\right)$.

Proof. By Lemma 4 , if $c \sim c^{\prime}$ and $c \in Z^{2}\left(\mathfrak{U}, \mathrm{G}_{t}\right), c^{\prime}$ belongs to $Z^{2}\left(\mathfrak{U}, \mathrm{G}_{t}\right)$. By definition, $c \sim c$. If $c \sim c^{\prime}$, we get

$$
\begin{aligned}
c_{i_{0} i_{1} i_{2}}=g_{i_{0} i_{1}} a_{i_{1} i_{2}}^{-1} g_{i_{0} i_{1}}^{-1} a_{i_{0} i_{1}{ }^{-1} c^{\prime}{ }_{i 0 i_{1} i_{2}} a_{i_{0} i_{2}}} \\
=a_{i_{0} i_{1}^{-1}}\left(a_{i_{0} i_{1}} g_{i_{0} i_{1}}\right) a_{i_{1} i_{2}}^{-1}\left(a_{i_{0} i_{1}} g_{i_{0} i_{1}}\right)^{-} c^{\prime} i_{0 i_{1} i_{2}}\left(a_{i_{0} i_{2}}{ }^{-1}\right)^{-1},
\end{aligned}
$$




$$
\begin{aligned}
& \left(\left(a_{i_{0} i_{1}} g_{i_{0} i_{1}}\right)^{-1} c^{\prime} i_{0} i_{1 i_{2}}\right)\left(\left(a_{i_{0} i_{2}} g_{i_{0} i_{2}}\right) a_{i_{2} i_{3}}{ }^{-1}\left(a_{i_{0} i_{2}} g_{i_{0} i_{2}}\right)^{-1}\right) \\
& =a_{i_{1} i_{2}}\left(g_{i_{0} i_{1}}{ }^{-1} c_{i_{0} i_{1} i_{2}} g_{i_{0} i_{2}}\right)\left(a_{i_{2} i_{3}}{ }^{-1} g_{i_{0} i_{2}}{ }^{-1}\right) a_{i_{0} i_{2}}{ }^{-1}, \\
& \left(\left(a_{i_{1} i_{2}} g_{i_{1} i_{2}}\right) a_{i_{2} i_{3}}{ }^{-1}\left(a_{i_{1} i_{2}} g_{i_{1} i_{2}}\right)^{-1}\right)\left(\left(a_{i_{0} i_{1}} g_{i_{0} i_{1}}\right)^{-1} c^{\prime}{ }_{i_{0} i_{1} i_{2}}\right) \\
& \left.=a_{i_{1} i_{2}}\left(g_{i_{1} i_{2}} a_{i_{2} i_{3}}{ }^{-1} g_{i_{1} i_{2}}{ }^{-1}\right)\left(g_{i_{0} i_{1}}{ }^{-1} c_{i_{0} i_{1} i_{2}}\right) a_{i_{0} i_{2}}\right) a_{i_{0} i_{2}}{ }^{-1} \text {. }
\end{aligned}
$$

Hence we have $c^{\prime} \sim c$. If $c \sim c^{\prime}$ and $c^{\prime} \sim c^{\prime \prime}$, we set

$$
c^{\prime \prime}{ }_{i_{0} i_{1} i_{2}}=b_{i_{0} i_{1}}\left(a_{i_{0} i_{1}} g_{i_{0} i_{1}}\right) b_{i_{1} i_{2}}\left(a_{i_{0} i_{1}} g_{i_{0 i} i_{1}}\right)^{-1} c^{\prime}{ }_{i_{0} i_{1} i_{2} i_{2}} b_{i_{0} i_{2}}{ }^{-1} \text {. }
$$

Then we get

$$
\begin{aligned}
c_{i_{0} i_{1} i_{2}}=b_{i_{0} i_{1}}\left(a_{i_{0} i_{1}} g_{i_{0} i_{1}}\right) b_{i_{1} i_{2}}\left(a_{i_{0} i_{1}} g_{i_{0} i_{1}}\right)^{-1} a_{i_{0} i_{1}} g_{i_{0} i_{1}}{ }^{-1} c_{i_{0} i_{1} i_{2}} a_{i_{0} i_{2}}{ }^{-1} b_{i_{0} i_{2}}{ }^{-1} \\
=\left(b_{i_{0} i_{1}} a_{i_{0} i_{1}}\right) g_{i_{0} i_{1}}\left(b_{i_{1} i_{2}} a_{i_{1} i_{2}}\right) g_{i_{0} i_{1}}{ }^{-1} c_{i_{0} i_{1} i_{2}}\left(b_{i_{0} i_{2}} a_{i_{0} i_{2}}\right)^{-1} .
\end{aligned}
$$

On the other hand, since

$$
\left(g^{\prime}{ }_{i_{0} i_{1}}{ }^{-1} c^{\prime}{ }_{i_{0} i_{1} i_{2}}\right)\left(g^{\prime}{ }_{i_{0} i_{2}} b_{i_{2} i_{3}} g^{\prime}{ }_{i_{0} i_{2}}{ }^{-1}\right)=\left(g^{\prime}{ }_{i_{1} i_{2}} b_{i_{2} i_{3}} g^{\prime}{ }_{i_{1} i_{2}}{ }^{-1}\right)\left(g^{\prime}{ }_{i_{0} i_{1}} c^{\prime}{ }_{i_{0} i_{1} i_{2}}\right), \quad g^{\prime}{ }_{i j}=a_{i j} g_{i j},
$$

we get

$$
a_{i_{1} i_{2}} g_{i_{0} i_{1}}{ }^{-1} c_{i_{0} i_{1} i_{2}} g_{i_{0} i_{2}} b_{i_{2} i_{3}} g_{i_{0} i_{2}}{ }_{1} a_{i_{0} i_{2}}{ }^{-1}=a_{i_{1} i_{2}} g_{i_{1} i_{2}} b_{i_{2} i_{3}} g_{i_{1} i_{2}}{ }^{-1} g_{i_{0} i_{1}}{ }^{-1} c_{i_{0} i_{1} i_{2}} a_{i_{0} i_{2}}{ }^{-1} \text {. }
$$

Hence we have

$$
\left(g_{i_{0} i_{1}}^{-1} c_{i_{0} i_{1} i_{2}}\right)\left(g_{i_{0} i_{2}} b_{i_{2} i_{3}} g_{i_{0} i_{2}}{ }^{-1}\right)=\left(g_{i_{1} i_{2}} b_{i_{2} i_{3}} g_{i_{1} i_{2}}^{-1}\right)\left(g_{i_{0} i_{1}}{ }^{-1} c_{i_{0} i_{1} i_{2}}\right) \text {. }
$$

Therefore we obtain

$$
\begin{aligned}
& \left(g_{i_{0} i_{1}}{ }^{-1} c_{i_{0} i_{1} i_{2}}\right)\left(g_{i_{0} i_{2}} b_{i_{2} i_{3}} a_{i_{2} i_{3}} g_{i_{0} i_{2}}{ }^{-1}\right) \\
& =\left(g_{i_{0} i_{1}}{ }^{-1} c_{i_{0} i_{1} i_{2}}\right)\left(g_{i_{0} i_{2}} b_{i_{2} i_{3}} g_{i_{0} i_{2}}{ }^{-1}\right)\left(g_{i_{0} i_{2}} a_{i_{2} i_{3}} g_{i_{0} i_{2}}{ }^{-1}\right) \\
& =\left(g_{i_{1} i_{2}} b_{i_{2} i_{3}} g_{i_{1 i_{2}}}{ }^{-1}\right)\left(g_{i_{0} i_{1}}{ }^{-1} c_{i_{0} i_{1} i_{2}}\right)\left(g_{i_{0} i_{1}} a_{i_{2} i_{3}} g_{i_{0} i_{2}}{ }^{-1}\right) \\
& \left.=g_{i_{1} i_{2}} b_{i_{2} i_{3}} g_{i_{1} i_{2}}{ }^{-1}\right)\left(g_{i_{1} i_{2}} a_{i_{2} i_{3}} g_{i_{1} i_{2}}{ }^{-1}\right)\left(g_{i_{0} i_{1}}{ }^{-1} c_{i_{0} i_{1} i_{2}}\right) \\
& =\left(g_{i_{1} i_{2}} b_{i_{2} i_{3}} g_{i_{1} i_{2}}{ }^{-1}\right)\left(g_{i_{0} i_{1}} c_{i_{0} i_{1} i_{2}}\right) .
\end{aligned}
$$

Hence $c^{\prime \prime} \sim c$, Therefore we have (i). (ii) follows from the following calculation:

$$
\begin{aligned}
& \left(h_{i_{0}} a_{i_{0} i_{1}} h_{i_{0}}{ }^{-1}\right)\left(h_{i_{0}} g_{i_{0} i_{1}} h_{i_{1}}^{-1}\right)\left(h_{i_{1}} a_{i_{1} i_{2}} h_{i_{1}}^{-1}\right)\left(h_{i_{0}} c_{i_{0} i_{1} i_{2}} h_{i_{0}}^{-1}\right) .\left(h_{i_{0}} a_{i_{0} i_{2}}{ }^{-1} h_{i_{0}}^{-1}\right) \\
& =h_{i_{0}}\left(a_{i_{0} i_{1}} g_{i_{0} i_{1}} a_{i_{1} i_{2}} g_{i_{0} i_{1}}^{-1} c_{i_{0} i_{1} i_{2}} a_{i_{0} i_{2}}{ }^{-1}\right) h_{i_{0}}{ }^{-1} \\
& \left(\left(h_{i_{1}} g_{i_{1} i_{0}} h_{i_{0}}^{-1}\right)\left(h_{i_{0}} c_{i_{0} i_{1} i_{2}} h_{i_{0}}^{-1}\right)\right)\left(\left(h_{i_{0}} g_{i_{0} i_{2}} h_{i_{2}}{ }^{-1}\right)\left(h_{i_{2}} a_{i_{2} i_{3}} h_{i_{2}}^{-1}\right) . \quad\left(h_{i_{2}} g_{i_{2} i_{0}} h_{i_{0}}^{-1}\right)\right) \\
& =h_{i_{1}}\left(\left(g_{i_{0} i_{1}}{ }^{-1} c_{i_{0} i_{1} i_{2}}\right)\left(g_{i_{0} i_{2}} a_{i_{2} i_{1}} g_{i_{0} i_{2}}{ }^{-1}\right)\right) h_{i_{0}}{ }^{-1} \text {, } \\
& \left(\left(h_{i_{1}} g_{i_{1} i_{2}} h_{i_{2}}{ }^{-1}\right)\left(h_{i_{2}} a_{i_{2} i_{3}} h_{{ }_{2}}^{{ }^{-1}}\right)\left(h_{i_{2}} g_{i_{2} i_{1}} h_{i_{1}}{ }^{-1}\right)\right)\left(\left(h_{i_{1}} g_{i_{1} i_{0}} h_{i_{0}}{ }^{-1}\right) .\left(h_{i_{0}} c_{i_{0} i_{1} i_{2}} h_{i_{0}}^{-1}\right)\right) \\
& =h_{i_{1}}\left(\left(g_{i_{1} i_{2}} a_{i_{2} i_{3}} g_{i_{1} i_{2}}{ }^{-1}\right)\left(g_{i_{0} i_{1}}^{-1} c_{i_{0} i_{1} i_{2}}\right)\right) h_{i_{0}}{ }^{-1} \text {. }
\end{aligned}
$$

Definition 23. We denote $\mathrm{H}^{2}\left(\mathfrak{H}, \mathrm{G}_{i}\right)$ (resp. $\left.\mathrm{H}^{2}\left(\mathfrak{H}, \mathrm{G}_{d}\right)\right)$ the quotient set of $\mathrm{Z}^{2}(\mathfrak{H}$, $\left.\mathrm{G}_{t}\right)$ (resp. $\left.\mathrm{Z}^{2}\left(\mathfrak{H}, \mathrm{G}_{d}\right)\right)$ by the relation of being cohomologous and admissible actions of the elements of $\mathrm{C}^{\circ}\left(\mathfrak{H}, \mathrm{G}_{d}\right)$.

Let $\mathfrak{B}=\left\{V_{j} \mid j \in \mathbb{J}\right\}$ be a refinement of $\mathfrak{U}=\left\{U_{i} \mid i \in \mathbb{I}\right\}$ and let $\tau, \tau^{\prime}: \mathbf{J}>\mathbb{I}$ the maps suchthat $V_{j} \subset U_{\tau(j) \wedge} U_{\tau^{\prime}(j)}$. We denote $\tau(j)=i$ and $\tau^{\prime}(j)=i^{\prime}$. We set 


$$
a_{i_{0} i_{1}}=c_{i_{0} i_{0} i_{1}} c_{i_{0} i_{1} i_{1}}{ }^{-1}
$$

Then we get

$$
\begin{aligned}
& a_{i_{0} i_{1}} g_{i_{0} i_{1}} a_{i_{1} i_{2}} g_{i_{0} i_{1}}{ }^{-1} c_{i_{0} i_{1} i_{2}} a_{i_{0} i_{2}}{ }^{-1} \\
& =c_{i_{0} i_{0}{ }^{\prime} i_{1}{ }^{\prime}} c_{i_{0} i_{1} i_{1}{ }^{\prime}}{ }^{-1} g_{i_{0} i_{1}} \quad c_{i_{1} i_{1} i_{2}{ }^{\prime}} c_{i_{1} i_{2} i_{2}{ }^{\prime}}{ }^{-1} g_{i_{0} i_{1}}{ }^{-1} c_{i_{0} i_{1} i_{2}} c_{i_{0} i_{2} i_{2}{ }^{\prime}} c_{i_{0} i_{0}{ }^{\prime} i_{2}{ }^{\prime}}{ }^{-1} \text {. }
\end{aligned}
$$

Since $c \in Z^{2}\left(\mathfrak{u}, \mathrm{G}(t)\right.$ or $\in Z^{2}\left(\mathfrak{l}, \mathrm{G}_{d}\right)$, we have

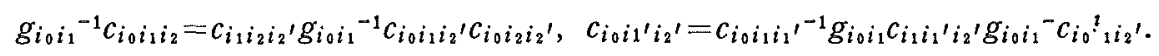

Hence we have

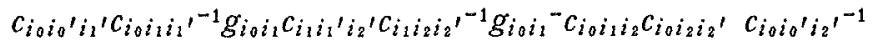

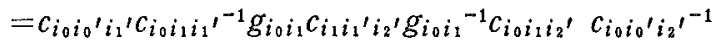

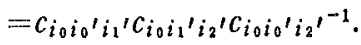

There fore we have

$$
a_{i_{0} i_{1}} g_{i_{0} i_{1}} a_{i_{1} i_{2}} g_{i_{0} i_{1}}^{-1} c_{i_{0} i_{1} i_{2}} a_{i_{0} i_{2}}{ }^{-1}=g_{i_{0} i_{0}} c_{i_{0} i_{i_{1}}^{\prime} i_{2}{ }^{\prime}} g_{i_{0} i_{0}}{ }^{-1} .
$$

Since $g=\left\{g_{i i}\right\}$ is $c$-admissible by Lemma 3 (ii)', this shows $\tau^{*}(c)$ and $\tau^{\prime *}(c)$ give the same element in $\mathrm{H}^{2}\left(M, \mathrm{G}_{t}\right)$ (or $\mathrm{H}^{2}\left(M, \mathrm{G}_{d}\right)$ ).

Definition 24. We set $\lim \left\{\mathrm{H}^{2}\left(\mathfrak{H}, \mathrm{G}_{t}\right) \mid \tau=\mathrm{H}^{2}\left(M, \mathrm{G}_{t}\right)\right\}$ and $\lim \left\{\mathrm{H}^{2}\left(\mathfrak{H}, \mathrm{G}_{d}\right) \mid \tau=\mathrm{H}^{2}\right.$ $\left.\left(M, \mathrm{G}_{d}\right)\right\}$.

Note. These cohomology sets are not defined absolutely from the sheaves $G_{t}$ and $\mathrm{G}_{d}$. Therefore the notations $\mathrm{H}^{2}\left(M, \mathrm{G}_{t}\right)_{\mathrm{G} d}$ and $\mathrm{H}^{2}\left(M, \mathrm{G}_{d}\right)_{\mathrm{G} d}$ are more exact. But we do not use these notations.

7. Theorem 2. There are maps $\delta=\delta_{0}: \mathrm{H}^{0}\left(M, \mathscr{K}^{1}\right) \rightarrow \mathrm{H}^{1}\left(M, \mathrm{G}_{t}\right)$ and $\delta=\delta_{1}: \mathrm{H}^{1}(M$, $\left.\mathscr{A}^{1}\right) \rightarrow \mathrm{H}^{2}\left(M, \mathrm{G}_{t}\right)$ and the following sequence is exact.

$$
\begin{aligned}
& 0 \longrightarrow \mathrm{H}^{0}\left(M, \mathrm{G}_{t}\right) \stackrel{i}{\longrightarrow} \mathrm{H}^{0}\left(M, \mathrm{G}_{d}\right) \stackrel{\rho^{*}}{\longrightarrow} \mathrm{H}^{0}\left(M, \mathscr{K}^{1}\right) \stackrel{\delta}{\longrightarrow} \mathrm{H}^{1}\left(M, \mathrm{G}_{t}\right) \stackrel{i^{*}}{\longrightarrow} \\
& \longrightarrow \mathrm{H}^{1}\left(M, \mathrm{G}_{d}\right) \stackrel{\rho^{*}}{\longrightarrow} \mathrm{H}^{1}\left(M, \mathscr{M}^{1}\right) \stackrel{\delta}{\longrightarrow} \mathrm{H}^{2}\left(M, \mathrm{G}_{t}\right) \stackrel{i^{*}}{\longrightarrow} \mathrm{H}^{2}\left(M, \mathrm{G}_{d}\right) .
\end{aligned}
$$

Proof. The exactness of the first six terms of (21) has been known (cf. [3], [15], [21]). $\delta_{0}$ is defined by

$$
\delta_{0}(\theta)=\left\{h_{U} h_{V}^{-1}\right\}, h_{U}^{-1} d h_{U}=\theta \mid U, \theta \in \mathrm{H}^{0}\left(M, \mathscr{M}^{1}\right) .
$$

We define $\delta_{1}$ by

$$
\delta_{1}\left(\left\langle\omega_{i j}\right\rangle\right)=\left\{g_{i j} g_{j k} g_{i k}^{-1}\right\}, \omega_{i j}=g_{i j}{ }^{-1} d g_{i j} .
$$

By Proposition 1, (i) and Lemma 3, (iv), $c_{i j k}=g_{i j} g_{j k} g_{i k}{ }^{-1}$ belongs to $Z^{2}\left(\mathfrak{H}, G_{t}\right)$. If $\omega_{i j}=\rho\left(g_{i j}\right)=\rho\left(g_{i j}{ }^{\prime}\right)$, set $g_{i j}=a_{i j} g_{i j}, a_{i j}$ is a constant map. We set $c_{i j k}{ }^{\prime}=g_{i j}{ }^{\prime} g_{k i}{ }^{\prime}$. Then we have

$$
c_{i_{0} i_{1} i_{2}{ }^{\prime}}=a_{i_{0} i_{1}} g_{i_{0} i_{1}} a_{i_{1} i_{2}} g_{i_{0} i_{1}}{ }^{-1} c_{i_{0} i_{1} i_{2}} a_{i_{0} i_{2}}{ }^{-1}
$$


Since (20) holds by the note at the end of Lemma $4,\left\{c_{i j k}{ }^{\prime}\right\}$ is cohomologous to $\left\{c_{i j k}\right\}$. Hence $\delta_{1}$ is well defined by Lemma 5, (ii). Then we have $\operatorname{ker} \delta_{1}=\left\{\omega_{i j} \mid \omega_{i j}=\right.$ $\left.\rho\left(g_{i j}\right), g_{i j} g_{j k} g_{k i}=\mathrm{e}\right\}=$ image $\rho^{*}$. If $i^{*}(c)$ vanishes, we have

$$
c_{i j k}=a_{i j} g_{i j} a_{i k} g_{i j^{-1}} a_{i k^{-1}}=b_{i j} g_{j k} g_{k j} g_{j i} g_{k i}{ }^{-1} b_{i k}{ }^{-1}, b_{i j}=a_{i j} g_{i j} .
$$

Hence ker $i^{*}$ is contained in image $\delta$. Since image $\delta$ is contained in ker $i^{*}$ by definition, we have Theorem 2.

Note. If $M$ is a complex manifold, we can define the cohomology sets $\mathrm{H}^{2}(M$, $\left.\mathrm{G}_{t}\right)_{\omega}=\mathrm{H}^{2}\left(M, \mathrm{G}_{t}\right)_{\mathrm{G}_{\omega}}$ and $\mathrm{H}^{2}\left(M, \mathrm{G}_{\omega}\right)=\mathrm{H}^{2}\left(M, \mathrm{G}_{\omega}\right)_{\mathrm{G}_{\omega}}$ by the same way. Then we get the exact sequence

$$
\begin{aligned}
& 0 \longrightarrow \mathrm{H}^{0}\left(M, \mathrm{G}_{t}\right) \longrightarrow \mathrm{H}^{0}\left(M, \mathrm{G}_{\omega}\right) \longrightarrow \mathrm{H}^{0}\left(M, \mathscr{H}^{1}{ }\right) \longrightarrow \mathrm{H}^{1}\left(M, \mathrm{G}_{t}\right) \longrightarrow \mathrm{H}^{1}\left(M, \mathrm{G}_{\omega}\right) \longrightarrow \\
& \longrightarrow \mathrm{H}^{1}\left(M, \mathscr{M}{ }^{1}\right) \longrightarrow \mathrm{H}^{2}\left(M, \mathrm{G}_{t}\right)_{\omega} \longrightarrow \mathrm{H}^{2}\left(M, \mathrm{G}_{\omega}\right)_{\omega} .
\end{aligned}
$$

Here, $\mathrm{H}^{2}\left(M, \mathrm{G}_{t}\right)_{\omega}$ may differ from $\mathrm{H}^{2}\left(M, \mathrm{G}_{t}\right)$.

If $n=1$, that is $\mathrm{G}=\mathrm{C}^{*}, \mathscr{H}^{1}$ becomes $\bar{\Phi}^{1}$, the sheaf of germs of closed 1 -forms over $M$ and we have $\mathrm{H}^{1}\left(M, \mathscr{M}^{1}\right)=\mathrm{H}^{2}(M, \mathbb{C}), \mathrm{H}^{1}\left(M, \mathrm{G}_{d}\right)=\mathrm{H}^{2}(M, \mathbb{Z})$ and $\mathrm{H}^{2}\left(M, \mathrm{G}_{d}\right)$ $=\mathrm{H}^{3}(M, \mathbb{Z})$. The exact sequence $(21)$ is rewritten as the following exact sequence

$$
\begin{aligned}
& 0 \longrightarrow \mathrm{H}^{0}\left(M, \mathbf{C}^{*}\right) \longrightarrow \mathrm{H}^{0}\left(M, \mathbf{C}^{*}{ }_{d}\right) \longrightarrow \mathrm{H}^{0}\left(M, \phi^{1}\right) \longrightarrow \mathrm{H}^{1}\left(M, \mathrm{C}^{*}\right) \longrightarrow \mathrm{H}^{2}(M, \mathbb{Z}) \longrightarrow \\
& \longrightarrow \mathrm{H}^{2}(M, \mathrm{C}) \stackrel{\exp ^{*}}{\longrightarrow} \mathrm{H}^{2}\left(M, \mathrm{C}^{*}\right) \longrightarrow \mathrm{H}^{3}(M, \mathbb{Z}) .
\end{aligned}
$$

This comes from the commutativity of the following diagram of sheaves

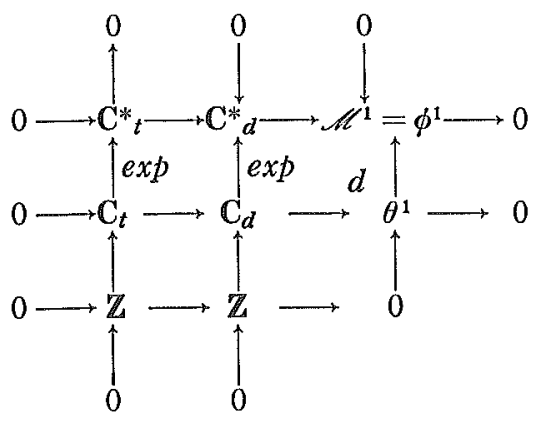

The corresponding diagramfor $\mathrm{GL}(n, \mathrm{C}), n \geqq 2$, takes the following form

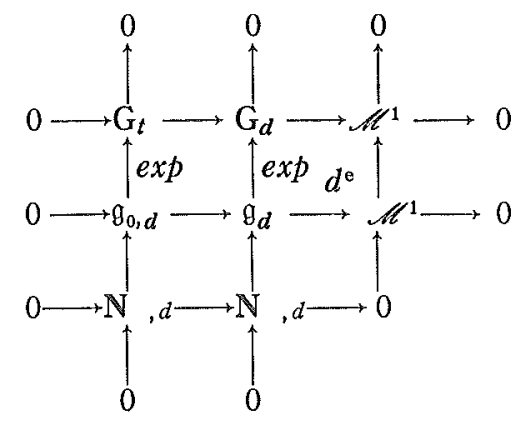


Here $\mathfrak{B}_{0, d}$ and $\mathbb{N}, d$ are defined as kernel sheaves of the maps $d^{\mathrm{e}}$ and exp. Detailed definitions of this diagram and related cohomology sets are given in [9].

8. We denote the natural map from $Z_{d}^{0}\left(M, \mathscr{K}^{2}\right)$ onto $\mathrm{H}^{2}{ }_{d R}(M, \theta)$ by $d R$. Then $\delta \iota d R$ maps $Z_{d}^{0}\left(M, \mathscr{M}^{2}\right)$ into $\mathrm{H}^{2}\left(M, \mathrm{G}_{t}\right)$. By Theorems 1 , 2, we have

Proposition 2. $\Theta=\left\{\Theta_{i}\right\} \in Z_{d}^{0}\left(M, \mathscr{M}^{2}\right)$ is realized as a curvature form of a Gbundle over $M$ if and only if $\delta, d R(\Theta)=0$.

Corollary. Let $\left\{M_{\mathrm{G}}, \pi, M\right\}$ be a principal G-bundle over $M$ and $\theta$ a matrix valued 1-form. Then $\Theta \in \mathrm{Z}_{d}^{0}\left(M, \mathscr{M}^{2}\right)$ can be written as

$$
\pi^{*}(\Theta)=d \theta+\theta \wedge \theta
$$

if and only if $\delta_{t} d R(\Theta)=0$. Here $M_{\mathrm{G}}$ depends on $\Theta$.

Proof. If (23) holds, $\Theta$ is realized as a curvature form. Hence we have the necessity. If $\Theta$ is a curvature form of $\xi=\left\{g_{i j}\right\}$ with the associated principal bundle $\left\{M_{\mathrm{G}}, \pi, M\right\}$, we have

$$
\pi^{*}(\Theta) \mid \pi^{-1}\left(U_{i}\right)=h_{i}^{-1} \theta_{i} h_{i} \pi^{*}\left(g_{i j}\right)=h_{i} h_{j}^{-1} .
$$

Since $\Theta$ is a curvature form, we can set $\Theta_{i}=d \theta_{i}{ }^{\prime}+\theta_{i}{ }^{\prime} \wedge \theta_{i}{ }^{\prime}$. Then we have $\theta$ to set

$$
\theta \mid \pi^{-1}\left(U_{i}\right)=h_{i}^{-1}\left(\theta_{i}^{\prime}+d h_{i} h_{i}^{-1}\right) h_{i} .
$$

Note 1. Usual de Rham groups are the obstructions of global solvabilities of the equations $d \varphi=\phi, \operatorname{deg} \psi=1,2, \ldots$, From this point of view, $\mathrm{H}^{1}\left(M, \mathscr{M}^{1}\right)$ is the obstruction of global solvability of the equation

$$
d^{\mathrm{e}} \theta=\Theta, \Theta=\left\{\Theta_{i}\right\}, \quad \theta=\left\{\theta_{i}\right\}, \quad \theta_{j}=g_{j i}\left(\theta_{i}\right), \Theta_{j}=g_{j i}\left(\Theta_{i}\right)
$$

where $\left\{g_{i j}\right\}$ belongs to $\mathrm{C}_{c}{ }_{c}\left(U, \mathrm{G}_{d}\right)$.

Note 2. If $d^{\mathrm{e}} \theta=d^{\mathrm{e}} \theta^{\prime}$, set $\theta^{\prime}=\theta+\eta, \eta$ satifies the equation

$$
d \eta+\eta \wedge \eta+[\theta, \eta]=0 .
$$

Local solutions of this equation and the relation between gauge transformations are studied in $[10] \mathrm{cf}$. [20].

§3. Characteristic classes for the elemens of $\mathrm{H}^{1}\left(M, \mathscr{H}^{1}\right)$.

9. Lemma 6. Let $\Theta=\left\{\Theta_{i}\right\}$ be an elemen of $Z^{0} d\left(M, \mathscr{M}^{2}\right)$. Then $\operatorname{tr}\left(\Theta_{\wedge}{ }^{b} \wedge_{\wedge} \Theta\right)$ is a closed $2 p$-form on $M$ and its cohomology class is determined by $d R(\Theta)$ for any $p$.

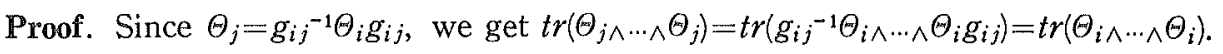
Hence $\operatorname{tr}\left(\Theta_{\wedge \cdots \wedge} \Theta\right)$ defines a global form on $M$. Since $\Theta_{i}=d^{\mathrm{e}} \theta_{i}$, we have the Bianchi identity $d \Theta=[\Theta, \theta]$. Then, since

$$
\operatorname{tr}\left(\stackrel{r-1}{\Theta_{\wedge} \ldots \Theta_{\wedge}[\theta,}, \Theta\right] \wedge \Theta_{\wedge \cdots \wedge}^{p-r}(\Theta)=0,
$$


we get

$$
d\left(t r\left(\Theta_{\wedge \cdots \wedge}^{p} \Theta\right)\right)=\sum_{r=1}^{p} \operatorname{tr}\left(\Theta_{\wedge \cdots}^{r-1} \Theta_{\wedge} d \Theta_{\wedge} \Theta_{\wedge \cdots \wedge}^{p-r} \Theta\right)=0 .
$$

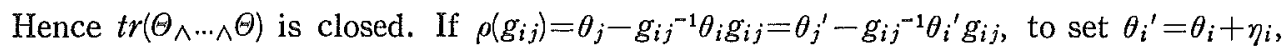
we have

$$
\begin{aligned}
& \theta_{j}=g_{i j}{ }^{-} \theta_{i} g_{i j}, \\
& d^{\mathrm{e}}\left(\theta_{i}{ }^{\prime}\right)=\theta_{i}+d \theta_{i}+\eta_{i \wedge} \eta_{i}+\left[\theta_{i}, \eta_{i}\right] .
\end{aligned}
$$

Then, since $\operatorname{tr}\left(\eta_{i} \wedge \eta_{i}\right)=\operatorname{tr}\left[\theta_{i}, \eta_{i}\right]=0$, we get

$$
\operatorname{tr}\left(d^{\mathrm{e}}\left(\theta_{i}{ }^{\prime}\right)_{\wedge \cdots \wedge} d^{\mathrm{e}}\left(\theta_{i}{ }^{\prime}\right)\right)=\operatorname{tr}\left(\left(\theta_{i}+d \eta_{i}\right)_{\wedge \cdots \wedge}\left(\theta_{i}+d \eta_{i}\right)\right) .
$$

By (25), to set $\phi_{i, 1}=\theta_{i}$ and $\phi_{i,-1}=d \eta_{i}$, we have

$$
\operatorname{tr}\left(\phi_{j}, \quad \varepsilon_{1 \wedge \cdots} \phi_{j, \varepsilon_{p}}\right)=\operatorname{tr}\left(\phi_{i, \varepsilon_{1} \wedge \cdots \wedge} \phi_{i, \varepsilon_{p}}\right), \quad \varepsilon_{k}= \pm 1
$$

Then, since

$$
\begin{aligned}
& \operatorname{tr}\left(\Theta_{i \wedge \cdots \wedge}^{r-1} \Theta_{i \wedge} d \eta_{i \wedge} \phi_{i, \varepsilon r+1 \wedge \cdots \wedge} \phi_{i, \varepsilon_{p}}\right) \\
& \quad \stackrel{r-1}{ } d\left(\operatorname{tr}\left(\Theta_{i \wedge \cdots \wedge} \Theta_{i \wedge} \phi_{i, \varepsilon_{r+1} \wedge \cdots \wedge} \phi_{i}, \varepsilon_{p}\right)\right), \quad r \geqq 1
\end{aligned}
$$

$\operatorname{tr}\left(d^{\mathrm{e}}\left(\theta_{i}{ }^{\prime}\right)_{\wedge \cdots \wedge}{ }^{D} d^{\mathrm{e}}\left(\theta_{i}{ }^{\prime}\right)\right)$ is cohomologous to $\operatorname{tr}\left(\Theta_{\wedge \cdots \wedge}{ }^{D} \cdots\right)$. Hence we have Lemma.

Corollary. If $\Theta \in Z^{0} d\left(M, \mathscr{H}^{2}\right)$, the coefficients of det $(\mathbb{I}+(t / 2 \pi \sqrt{-1}) \Theta)$ are closed anp their de Rham classes are determined by \& $d r(\Theta)$.

Definition 25. Let $\langle\omega\rangle$ be an element of $\mathrm{H}^{1}\left(M, \mathscr{M}^{1}\right), \Theta$ an element of $\mathrm{Z}^{0}{ }_{d}(M$, $\left.\mathscr{H}^{2}\right)$ such that,$d R(\Theta)=\langle\omega\rangle$. Then we define the $p$-th characteristic class $c^{p}(\langle\omega\rangle)$ of $\langle\omega\rangle$ by

$$
\begin{aligned}
& c^{p}(\langle\omega\rangle)=\left\langle\varphi_{p}\right\rangle, \text { the de Rham class of } \varphi_{p}, \\
& \operatorname{det}\left(\mathbb{I}+\frac{t}{2 \pi \sqrt{-1}} \Theta\right)=\mathbb{I}+\varphi_{1} t+\cdots+\varphi_{p} t^{p}+\cdots+\varphi_{n} t^{n} .
\end{aligned}
$$

Similarly, we define the $p$-th Chern character of $\langle\omega\rangle$ by

$$
\operatorname{ch}(\langle\omega\rangle)=\left(\frac{\sqrt{-1}}{2 \pi}\right)^{p} \frac{1}{p !} r t\left(\Theta_{\wedge \cdots \wedge}^{p} \Theta\right)
$$

Proposition 3. (i) $c^{p}(\langle\omega\rangle)$ and $c h^{p}(\langle\omega\rangle)$ aredetermined by $\langle\omega\rangle$.

(ii) Let $\xi$ be a $\mathrm{G}$-bundle with the $p$-th Chern class $c^{p(\xi)}$ and the $p$-th Chern character $\left.\operatorname{ch}^{p}(\xi), i^{*}: \mathrm{H}^{2 p} M, \mathbb{Z}\right) \rightarrow \mathrm{H}^{2 p}(M$, C) the map induced from the inclusion $i: \mathbb{Z} \rightarrow$ C. Then we have

$$
c^{p}\left(\rho^{*}(\xi)\right)=i^{*}\left(c^{p}(\xi)\right),
$$


$(26)^{\prime}$

$$
\operatorname{ch}^{p}\left(\rho^{*}(\xi)\right)=\operatorname{ch}^{p}(\xi)
$$

(iii) If $\omega=\left\{\omega_{i j}\right\}$ and each $\omega_{i j}$ is an antihermitian matrix valued 1 -form, $c^{p}(\langle\omega\rangle)$ and $c h^{p}(\langle\omega\rangle)$ belong to $\mathrm{H}^{2 p}(M, \mathbb{R})$.

Proof. (i) follows from Lemma 6 and its Corollary. (ii) follows from the theorem of Chern ([12]) and the definition of the Chern character. If $\omega$ satisfies the assumption of (iii), there exists an antihermitian matrix valued $\Theta \in Z_{d}^{0}\left(M, \mathscr{K}^{2}\right)$ such that $\iota d R(\Theta)=\langle\omega\rangle$. Then, since $\operatorname{det}(\mathbb{I}+(t / 2 \pi \sqrt{-1)} \Theta)$ is a real form coefficients poly nomial, we have (iii).

Corollary. $\Theta \in Z^{0}{ }_{d}\left(M, \mathscr{M}^{2}\right)$ can not be realized as a curvature form of a $\mathrm{G}^{-}$ bundle over $M$ if $c^{p}(\Theta)$ is not an integral class for some $p$.

Note. For an element $\theta$ of $\mathrm{H}^{0}\left(M, \mathscr{M}^{1}\right)$, we have defined its characteristic class $\beta^{p}(\theta) \in \mathrm{H}^{2 p-1}(M$, C) by

$$
\beta^{p}(\theta)=\text { the de Rham class of } \frac{(-1)^{p-1}}{(2 \pi \sqrt{-1})^{p}} \operatorname{tr}\left(\begin{array}{c}
2 p-1 \\
\left(\theta_{\wedge \cdots \wedge} \theta\right)
\end{array}\right.
$$

([6], cf. [27]). The definitions of $\beta^{p}(\theta)$ and $\operatorname{ch}^{p}(\Theta)$ are parallel. Moreover, $\beta^{p}(\theta)$ comes from the $p$-th generator of $\mathrm{H}^{*}(\mathrm{G}, \mathbb{Z})$ and $c^{p}(\Theta)$ comes from the $p$-th generator of $\mathrm{H}^{*}(\mathrm{BG}, \mathbb{Z})$.

10. Example 1. If $\lambda$ is a complex number, then we have

$$
d^{\mathrm{e}}(\lambda \theta)=\lambda d^{\mathrm{e}} \theta+\left(\lambda^{2}-\lambda\right) \theta_{\wedge} \theta .
$$

Hence we have

$$
\operatorname{tr}\left(d^{\mathrm{e}}(\lambda \theta)_{\wedge \cdots \wedge} \stackrel{p}{\mathrm{e}} d^{\mathrm{e}}(\lambda \theta)=\lambda^{p} \operatorname{tr}\left(d^{\mathrm{e}} \theta_{\wedge \cdots \wedge}^{p} d^{\mathrm{e}} \theta\right) .\right.
$$

Therefore, if $\operatorname{det}\left(\mathbb{I}+(t / 2 \pi \sqrt{-1}) d^{\mathrm{e}} \theta\right)=\mathbb{I}+\varphi_{1} t+\cdots+\varphi_{n} t^{n}$, we have

$$
\operatorname{det}\left(\mathbb{I}+\frac{t}{2 \pi \sqrt{-1}} d^{\mathrm{e}}(\lambda \theta)\right)=\mathbb{I}+\lambda \varphi_{1} t+\cdots+\lambda^{p} \varphi_{p} t^{p}+\cdots+\lambda^{n} \varphi_{n} t^{n} .
$$

On the other hand, if $g$ is a smooth G-valued function such that

$$
(d g) g=g(d g)
$$

and $g^{\lambda}$ is defined, then

$$
\rho\left(g^{\lambda}\right)=\lambda \rho(g) \text {. }
$$

Hence if $\xi=\left\{g_{i j}\right\} \in \mathrm{H}^{1}\left(M, \mathrm{G}_{d}\right)$ satisfies (28) for any $g_{i j}$ and for a fixed complex number $\lambda, g_{i j}{ }^{\lambda}$ is defined for each $i, j$, we get

$$
\rho\left(g_{i j}{ }^{\lambda}\right)=\left(\lambda \theta_{j}\right)-g_{i j^{-1}}\left(\lambda \theta_{i}\right) g_{i j}, \quad \theta_{j}-g_{i j^{-1}} \theta_{i} g_{i j}=\rho\left(g_{i j}\right) .
$$

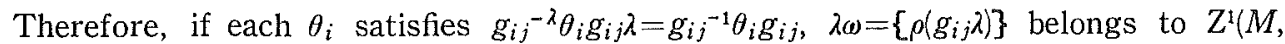
$\left.\mathscr{M}^{1}\right)$. Here $\boldsymbol{m}=\left\{\rho\left(g_{i j}\right)\right\}$. Then by (27), we have 


$$
c^{p}(\langle\lambda \omega\rangle)=\lambda_{p} c^{p}(\langle\omega\rangle)
$$

Example 2. Let $M$ be the $m$-dimensional complex projective space and $\left\{\mu_{1}, \ldots\right.$ ., $\left.\mu_{m}\right\}$ an arbitrary set of $m$ complex numbers. We set

$$
\begin{gathered}
\omega_{i j}=\left(\begin{array}{cc}
\lambda_{1}\left(\frac{d z_{j}}{z_{j}}-\frac{d z_{i}}{z_{i}}\right) & \\
\cdots & \\
\cdots & \\
0 & \cdots \\
& \lambda_{m}\left(\frac{d z_{j}}{z_{j}}-\frac{d z_{i}}{z_{i}}\right)
\end{array}\right), \\
\prod_{i=1}^{m}\left(1+\lambda_{i} t\right)=1+\mu_{1} t+\cdots+\mu_{m} t^{m}
\end{gathered}
$$

Then $\omega=\left\{\omega_{i j}\right\}$ defines an element $\langle\omega\rangle$ of $\mathrm{H}^{1}\left(M, \mathscr{M}^{1}\right)$ such that

$$
c^{p}(\langle\oplus\rangle)=\lambda_{p} \mathrm{e}^{p}, \mathrm{e}^{p} \text { is the generator of } \mathrm{H}^{2 p}(M, \mathbb{Z}) .
$$

Note. Let $\mathrm{U}=\mathrm{U}(n)$ be the unitary group, $\mathfrak{h}$ its Lie algebra. Then the sequence $0 \rightarrow \mathrm{U}_{t} \rightarrow \mathrm{U}_{d^{1}} \rightarrow \mathscr{K}_{\mathfrak{h}^{1} \rightarrow 0}$ is exact. Here $\mathscr{K}_{\mathfrak{h}^{1}}$ is the sheaf of germs of $\mathfrak{b}$-valued integrable connections. By this sequence, we get the following cohomology exact sequence

$$
\begin{aligned}
& 0 \longrightarrow \mathrm{H}^{0}\left(M, \mathrm{U}_{t}\right) \longrightarrow \mathrm{H}^{0}\left(M, \mathrm{U}_{d}\right) \longrightarrow \mathrm{H}^{0}\left(M, \mathscr{M}_{\mathfrak{l}^{1}}\right) \longrightarrow \mathrm{H}^{1}\left(M, \mathrm{U}_{t}\right) \longrightarrow \\
& \longrightarrow \mathrm{H}^{1}\left(M, \mathrm{U}_{d}\right) \longrightarrow \mathrm{H}^{1}\left(M, \mathscr{H}_{\mathfrak{l}^{1}}\right) \longrightarrow \mathrm{H}^{2}\left(N, \mathrm{U}_{t}\right) \longrightarrow \mathrm{H}^{2}\left(M, \mathrm{U}_{d}\right) .
\end{aligned}
$$

In this sequence, we know $\mathrm{H}^{1}\left(M, \mathrm{G}_{d}\right)=\mathrm{H}^{1}\left(M, \mathrm{U}_{d}\right)$. But example 2 and Proposition 3, (iii) show $\mathrm{H}^{1}\left(M, \mathscr{H}^{1}\right)$ differes from $\mathrm{H}^{1}\left(M, \mathscr{H}_{\mathfrak{h}^{1}}\right)$ in general. We also know $\mathrm{H}^{1}(M$, $\left.\mathrm{G}_{t}\right)$ differs from $\mathrm{H}^{1}\left(M, \mathrm{U}_{t}\right)$. For example, denote $\sigma$ the generator of $\pi_{1}\left(\mathrm{C}^{*}\right)$, the representation $\chi_{o}=\left[\begin{array}{cc}1 & 2 \pi \sqrt{-1} \\ 0 & 1\end{array}\right]$ defines an element of $\mathrm{H}^{1}\left(\mathrm{C}^{*}, \mathrm{GL}(2, \mathrm{C})\right)$ which is not in $\mathrm{H}^{1}\left(\mathrm{C}^{*}, \mathrm{U}(2)\right)$.

11. If $c=c_{i j k}$ belongs to $Z^{2}\left(\mathfrak{H}, \mathrm{G}_{t}\right)$ resp. to $\left.Z^{2}\left(\mathfrak{H}, \mathrm{G}_{d}\right)\right)$, det $c=\left\{\right.$ det $\left.c_{i j k}\right\}$ belong to $Z^{2}\left(\mathfrak{U}, \mathbb{C}^{*}\right)$ (resp. to $Z^{2}\left(\mathfrak{u}, \mathbb{C}_{d}^{*}\right)$ ). Since the cohomology class of det $c$ is determined by the cohomology class of $c$, we define

$$
d^{\prime}(\langle c\rangle)=\langle\operatorname{det} c\rangle .
$$

By definition, $d^{1}(\langle c\rangle) \in \mathrm{H}^{2}\left(M, \mathbf{C}^{*}\right)\left(\operatorname{resp} . \mathrm{H}^{2}\left(M, \mathbf{C}_{* d}\right)\right)$ and we have

$$
\exp ^{*}\left(c^{1}(\langle\omega\rangle)\right)=d^{1}(\delta(\langle\omega\rangle))
$$

To treat higher dimensional chatacteristic classes for $\langle c\rangle$, we set $a_{i_{0} i_{1} i_{2}}=$ $(1 / 2 \pi \sqrt{-1}) \log \left(c_{i_{0} i_{1} i_{2}}\right)$. Then $a=\left\{a_{i_{0} i_{1} i_{2}}\right\}$ belongs to $\mathbb{C}^{2}(\mathfrak{H}, \quad$ g). $a$ is not uniquəly determined from $c$. But we can construct appropriate cohomology theory with 
coefficients in $\mathfrak{g}_{0, d}$ and $g_{d}$. To use this theory, if $a$ satisfies

$$
\operatorname{tr}(\delta a \cup \cdots \cup \delta a) \in Z^{3 p}(\mathfrak{H}, \mathbb{Z}),
$$

We can define higher order characteristic classes $d^{f}\left(\langle c) \in \mathrm{H}^{3 p-1}\left(M, \mathrm{C}^{*}\right)\right.$ and $e^{p}(c) \in$ $\mathrm{HH}^{3 p}(M, \mathbb{Z}), c \in \mathrm{H}^{2}\left(M, \mathrm{G}_{d}\right)$. For details, see [9].

Note. Researches on 3-dimensional theory suggest the possibility of the existence of a matrix valued 3-form (defined relative to gauge and potential) $\Phi=\mathscr{\Phi}(c)$ such that

$$
i^{*}\left(e^{p}(c)\right)=\text { the de Rham class of } \operatorname{tr}\left(\phi_{\wedge \cdots \wedge} \cdots \phi\right) .
$$

If this is true, $e^{2 p}(c)$ is a torsion class for any $p$, although it exists as an non zero class.

12. Let $Y$ be a closed subset of $M$. Then we have the following commutative diagram with exact lines

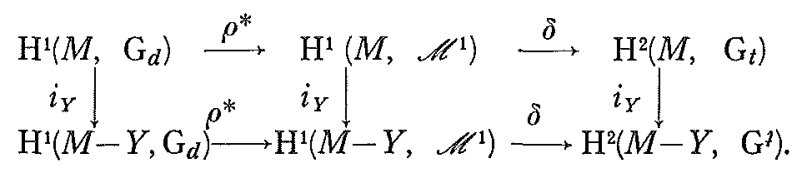

Hence we have

Lemma 7. Let $\langle\omega\rangle$ be an element of $\mathrm{H}^{1}\left(M, \mathscr{M}^{1}\right)$. Then $i_{Y}(\langle\omega\rangle)$ is in $\rho^{*}$-image ifand only if $i_{Y} \delta(\langle\omega\rangle)$ is equal to 0 .

Corollary. Let $\Theta \in \mathrm{Z}^{0}{ }_{d}\left(M, \mathscr{H}^{2}\right)$. On the tatal space of some G-bundle over $M-$ $Y, \Theta$ is written

$$
\pi^{*}(\Theta)=d \theta+\theta \wedge \theta
$$

if $i_{Y} \delta \iota d R(\Theta)=0$.

We write $r$ the codimension of $\mathrm{Y}$ and assume $r \geqq 2$. We also assume $M$ and $Y$ are cooriented and $Y$ is a smooth submanifold (in the case $M$ is a smooth manifold) or a real analytic subvariety in the case $M$ is a real analytic manifold). Then we have the following commutative diagram with exact lines and columns

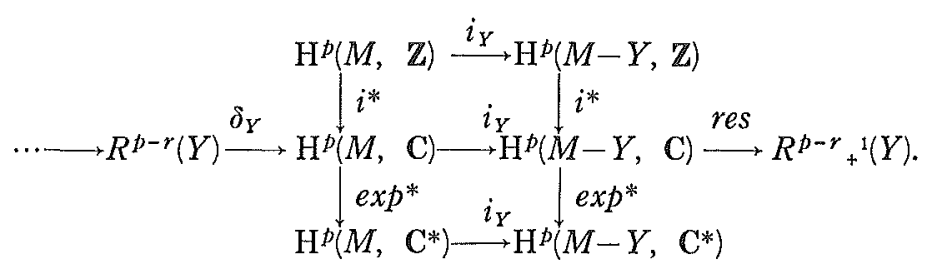

Here $R^{p}(Y)=\mathrm{H}^{p}(Y, \mathbf{C})$ if $Y$ is topologically non-singular $([4])$. By this diagram, we obtain 
Lemma 8. Let $c$ be an element of $\mathrm{H}^{p}(M, \mathrm{C})$. Then $i_{Y}(c)$ is in $i^{*}$-image if and only if $i_{Y} \exp ^{*}(c)=0$, and if $c$ is in $\delta_{Y^{-}}$image, $i_{Y} \exp ^{*}(c)=0$.

By Lemma 8, we have

Proposition 4. Let $\langle\omega\rangle$ be an element of $\mathrm{H}^{1}\left(M, \mathscr{M}^{1}\right)$ such that $i_{Y}(\langle\omega\rangle)$ is in $\rho^{*}$ image. Then we have

$$
i_{Y} \exp ^{*}\left(c^{p}(\langle\omega\rangle)\right)=0, \quad p \geqq 1 .
$$

(33) holds if we have

$$
c^{p}(\langle\omega\rangle)=\delta_{Y}\left(\left\langle\alpha_{p}\right\rangle\right),\left\langle\alpha_{p}\right\rangle \in R^{2 p-r}(Y) .
$$

Corollary. If $\pi^{*}(\Theta)=d^{\mathrm{e} \theta}$ on $(M-Y)_{\mathrm{G}^{\prime}}$ the total space of some principal bundle over $M-Y$, then

$$
i_{Y} \exp ^{*}\left(c^{p}(\Theta)\right)=0, \quad p \geqq 1 .
$$

(33)' holds if we have

$$
c^{p}(\Theta)=\delta_{Y}\left(\left\langle\alpha_{p}\right\rangle\right),\left\langle\alpha_{p}\right\rangle \in R^{2 p-r}(Y) .
$$

Note. we denote $\mathrm{G}_{d}[Y]$ and $\mathscr{M}^{1}[Y]$ the sheaves of germs of smooth Gvalued functions and integrable connections over $M$ with singularities on $Y$ (cf. [4)]. Then we have the following exact sequences of sheaves

$$
\begin{aligned}
& 0 \longrightarrow \mathrm{G}_{t} \stackrel{i_{y}}{\longrightarrow} \mathrm{G}_{d}[Y] \longrightarrow \rho\left(\mathrm{G}_{d}[Y]\right) \longrightarrow 0, \\
& 0 \longrightarrow \rho\left(\mathrm{G}_{d}[Y]\right) \longrightarrow \mathscr{M}^{1}[Y] \longrightarrow \operatorname{Res}_{\mathrm{G}, Y} \longrightarrow 0 .
\end{aligned}
$$

The stalk $\operatorname{Res}_{\mathrm{G}, Y, x}$ of $\operatorname{Res}_{\mathrm{G}, Y}$ at $x$ given by

$$
\operatorname{Res}_{\mathrm{G}, Y, x}=\lim \left[\delta\left(\mathrm{H}^{0}\left(U(x)-Y, \mathscr{M}^{1}\right)\right)\right] \text {. }
$$

Hence write $\pi_{1}(U-Y)_{x}$ the local fundamental group of $U-Y$ at $x, \operatorname{Res}_{\mathrm{G}, Y, x}$ is contained in Hom $\left(\pi_{1}(U-Y)_{x} \mathrm{G}\right)$ and therefore $\operatorname{Res}_{\mathrm{G}, Y}=0$ if $r \geqq 3$.

By (35), we have the following commutative diagram with exact lines

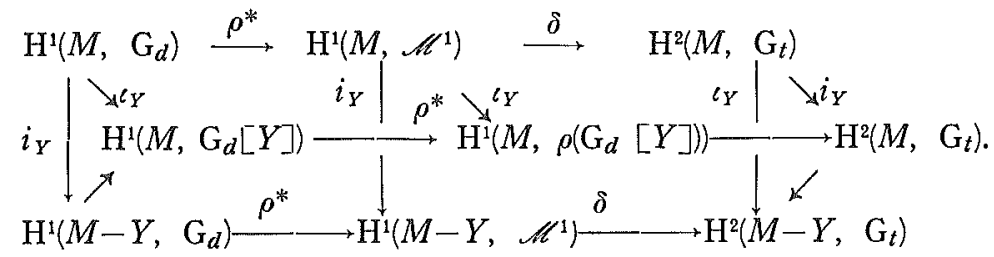

If $Y$ is smooth and $r$ is even, $\mathrm{H}^{1}\left(M, \mathrm{G}_{d}[Y]\right)=\mathrm{H}^{1}\left(M-Y, \mathrm{G}_{d}\right)$. In this case, $\iota_{Y}(\langle\omega\rangle)$ is not in $\rho^{*}$-image if $\langle\omega\rangle$ is not in $\rho^{*}$-image although $i_{Y}(\langle\omega\rangle)$ is in $\rho^{*}$-image. Therefore, if the equivalence of $i_{Y}(\langle\omega\rangle)$ and an element in $\rho^{*}$-image is given by $\left\{h_{i}\right\},\left\{h_{i}\right\}$ is not defined using open covering of $M-Y$ obtained to restrict an open covering of $M$ to $M-Y$. It also suggests $\mathrm{H}^{1}\left(M, \mathscr{M}^{1}[Y]\right)$ may differ from $\mathrm{H}^{1}(M-$ 
$\left.Y, \mathscr{H}^{1}\right)$

\section{\$4. The general case}

13. We fix a differential operator $D: \mathrm{C}^{\infty}(M, E) \rightarrow \mathrm{C}^{\infty}(M, F)$, where $E, F$ are complex vector bundles over $M$. We take a G-vector space $H$. Then $D_{i} \otimes 1_{H}: \mathrm{C}^{\infty}$ $\left(U_{i}, E \otimes H\right) \rightarrow \mathrm{C}^{\infty}\left(U_{i}, F \otimes H\right)$ is a differential operator on $U_{i}$. Here $D=\left\{D_{i}\right\}$ and $E$ and $F$ are both trivial on each $U_{i}$. By assumption, a smooth $\mathrm{G}$-valued function $g$ on $U_{i}$ acts on $\mathrm{C}^{\infty}\left(U_{i}, E \otimes H\right)$ and on $\mathrm{C}^{\infty}\left(U_{i}, F \otimes H\right)$. We set

$$
\rho_{D}(g)=g^{-1}\left(D_{i} \otimes 1_{H}\right) g-D_{i} \otimes 1_{H}
$$

If $\rho_{D}(g)=0, g$ is called a $c(D)$-class function ([6], [8]). The sheaf of germs of $c(D)$ -class G-valued functions is denoted by $\mathrm{G}_{c(D)} \cdot \rho_{D}\left(\mathrm{G}_{d}\right)$ is denoted by $\mathscr{M}^{1}{ }_{D}$ (in [6], this sheaf was denoted by $\left.L_{\mathrm{G}, D}\right)$. By definitions, we have the following exact sequence

$(1)_{D}$

$$
0 \longrightarrow \mathrm{G}_{c(D)} \stackrel{i}{\longrightarrow} \mathrm{G}_{d} \stackrel{D}{\longrightarrow} M^{1}{ }_{D} \longrightarrow 0 .
$$

By the definition of $\rho_{D^{\prime}}$ we have

$$
\rho_{D}(g h)=h^{-1} \rho_{D}(g) h+\rho_{D}(g), \rho_{D}(\mathrm{e})=0, \mathrm{e}=1_{H} .
$$

Hence we have

$$
\rho_{D}\left(g_{i j} g_{j k} g_{i k}{ }^{-1}\right)=g_{i k}\left(\rho_{D}\left(g_{j k}\right)-\rho_{D}\left(g_{i k}\right)+g_{j k}{ }^{-1} \rho_{D}\left(g_{i j}\right) g_{j k}\right) g_{i k}{ }^{-1} .
$$

By this formula, we can define the cohomology sets $\mathrm{H}^{1}\left(M, \mathscr{M}^{1}{ }_{D}\right)$ and $\mathrm{H}^{2}\left(M, G_{C(D)}\right)$ as follows;

Definitions $11 D$ and $21 D$. We set

$$
\begin{aligned}
& Z^{1}\left(\mathfrak{H}, \mathscr{M}^{1}{ }_{D}\right)=\left\{\left\{\omega_{i j}\right\} \in \mathrm{C}^{1}\left(\mathfrak{H}, \mathscr{M}^{1}{ }_{D}\right) \mid \delta_{\xi} \omega=0, \quad \omega=\rho_{D}(\xi), \quad \xi \in \mathrm{C}^{1} a\left(\mathfrak{H}, \mathrm{G}_{d}\right)\right\}, \\
& \mathrm{Z}^{2}\left(\mathfrak{H}, \mathrm{G}_{c(D)}\right)=\left\{c \in \mathrm{C}^{2}\left(\mathfrak{H}, G_{c(D)}\right) \mid \delta_{\xi} c=0, \text { for some } \xi \in C^{1}{ }_{c(D)}\left(\mathfrak{H}, \mathrm{G}_{d}\right)\right\} .
\end{aligned}
$$

Here $\mathrm{C}_{c(D)}^{1}\left(\mathfrak{H}, \mathrm{G}_{d}\right)$ is $\left\{\xi \in \mathrm{C}^{1} a\left(\mathfrak{U}, \mathrm{G}_{d}\right)\right\}(\delta \xi)_{i j k}$ is a $c(D)$-class fuction for anyi, $\left.j, k\right\}$.

Definitions $12 D$ and $22_{D}$. The cohomologous relations on $Z^{1}\left(\mathfrak{U}, \mathscr{H}^{1}{ }_{D}\right)$ and on $\mathrm{Z}^{2}\left(\mathfrak{H}, \mathrm{G}_{c(D)}\right)$ are defined as follows:

$$
\begin{aligned}
\left\{\omega_{i j}{ }^{\prime}\right\} \sim\left\{\omega_{i j}\right\} \text { if } \omega_{i j} j^{\prime}=h_{j}\left(\omega_{i j}-\rho_{D}\left(h_{j}\right)+g_{i j^{-1}} \rho_{D}\left(h_{i}\right) g_{i j}\right) h_{j}{ }^{-1} \\
\left\{h_{i}\right\} \in \mathrm{C}^{0}\left(\mathfrak{H}, \mathrm{G}_{d}\right), t_{i j k} \rho_{D}\left(h_{i}\right) t_{i j k}=\rho_{D}\left(h_{i}\right),
\end{aligned}
$$

and

$$
\begin{aligned}
& \left\{c_{i j k}\right\} \sim\left\{h_{i} c_{i j k} h_{i}^{-1}\right\}, \quad\left\{h_{i}\right\} \in \mathrm{C}^{0}\left(\mathfrak{H}, \mathrm{G}_{d}\right), \\
& \left\{c_{i j k}\right\} \sim\left\{a_{i j} g_{i j} a_{j k} g_{i j}{ }^{-1} c_{i j k} a_{i k}{ }^{-1}\right\}, \quad\left\{a_{i j}\right\} \in \mathrm{C}^{1}\left(\mathfrak{H}, \mathrm{G}_{c(D)}\right) .
\end{aligned}
$$

Here $\left\{a_{i j} g_{i j}\right\}$ belongs to $\mathrm{C}_{c(D)}^{1}\left(\mathfrak{H}, \mathrm{G}_{d}\right)$ and satisfies the following condition

$$
\left(g_{i_{0} i_{1}}{ }^{-1} c_{i_{0} i_{1} i_{2}}\right)\left(g_{i_{0} i_{2}} a_{i_{2} i_{3}} g_{i_{0} i_{2}}{ }^{-1}\right)=\left(g_{i_{1} i_{2}} a_{i_{2} i_{3} g_{i_{12}}}{ }^{-1}\right)\left(g_{i_{0} i_{1}}{ }^{-1} c_{i_{0} i_{1} i_{2}}\right) \text {. }
$$


$H^{1}\left(M, \mathscr{M}^{1}{ }_{D}\right)$ and $\left.\mathrm{H}^{2} M, \mathrm{G}_{c(D)}\right)$ are defined by these relations. Then we obtain

Theorem 2D. The following sequence is exact

$$
\begin{aligned}
& 0 \longrightarrow H^{0}\left(M, \mathrm{G}_{c(D)}\right) \stackrel{i}{\longrightarrow} \mathrm{H}^{0}\left(M, \mathrm{G}_{d}\right) \stackrel{\rho_{D}}{\longrightarrow} \mathrm{H}^{0}\left(M, \mathscr{M}^{1}{ }_{D} \longrightarrow \mathrm{H}^{1}\left(M, \mathrm{G}_{c(D)}\right) \stackrel{i^{*}}{\longrightarrow}\right. \\
& \mathrm{H}^{1}\left(M, \mathrm{G}_{d}\right) \stackrel{\rho_{D}{ }^{*}}{\longrightarrow} \mathrm{H}^{1}\left(M, \mathscr{A}^{1}{ }_{D}\right) \longrightarrow \mathrm{H}^{2}\left(M, \mathrm{G}_{c(D)}\right) \stackrel{i^{*}}{\longrightarrow} \mathrm{H}^{2}\left(M, G_{d}\right) .
\end{aligned}
$$

Example. If $D=\bar{\partial}, \mathrm{G}_{c\left(D_{1}\right)}$ is the sheaf of germs of holomorphic G-valued fuctions on $M$ and $\mathscr{M}_{D}$ is the sheaf of germs of matrix valued $(0,1)$-forms $\theta$ such that $\bar{\partial} \theta+\theta_{\wedge} \theta=0$ ([18]). If $\mathrm{G}=\mathrm{C}^{*},(21)_{D}$ reduces to

$$
\begin{aligned}
& 0 \longrightarrow \mathrm{H}^{0}\left(M, \mathbf{C}^{*}{ }_{\omega}\right) \longrightarrow \mathrm{H}^{0}\left(M, \mathrm{C}^{*}{ }_{d}\right) \longrightarrow \mathrm{H}^{0}\left(M, \phi^{0,1}\right) \longrightarrow \mathrm{H}^{1}\left(M, \mathbf{C}^{*}{ }_{\omega}\right) \longrightarrow \\
& \longrightarrow \mathrm{H}^{1}\left(M, \mathrm{C}^{*}{ }_{d}\right) \longrightarrow \mathrm{H}^{1}\left(M, \phi^{0,1}\right) \longrightarrow \mathrm{H}^{2}\left(M, \mathrm{C}^{*}{ }_{\omega}\right) \longrightarrow \mathrm{H}^{2}\left(M, \mathbf{C}^{*}{ }_{d}\right) .
\end{aligned}
$$

Here $\bar{\Phi}^{0,1}$ is the sheaf of germs of $\bar{\partial}$-closed $(0,1)$-forms. If $M$ is a compact Kaehler manifold, this sequence is rewritten to

$$
\begin{aligned}
& 0 \longrightarrow \mathrm{H}^{0}\left(M, \mathrm{C}^{*}{ }_{\omega}\right) \longrightarrow \mathrm{H}^{0}\left(M, \mathrm{C}^{*}{ }_{d}\right) \longrightarrow \mathrm{H}^{0}\left(M, \phi^{01}\right) \longrightarrow \mathrm{H}^{1}\left(M, \mathrm{C}^{*} \omega\right) \longrightarrow \\
& \longrightarrow \mathrm{H}^{2}(M, \mathbb{Z}) \longrightarrow \mathrm{H}^{0,2}(M, \mathrm{C}) \stackrel{\exp }{\longrightarrow} \mathrm{H}^{2}\left(M, \mathrm{C}^{*}{ }_{\omega}\right) \longrightarrow \mathrm{H}^{3}(M, \mathbb{Z}) .
\end{aligned}
$$

On the other hand, if $M$ is a Stein manifold, $i^{*}: \mathrm{H}^{1}\left(M, \mathrm{G}_{\omega}\right) \rightarrow \mathrm{H}^{1}\left(M, \mathrm{G}_{d}\right)$ is a $\operatorname{dijec}-$ tion. Hence the sequence $0 \rightarrow \mathrm{H}^{1}\left(M, \mathscr{M}^{1-}\right) \rightarrow \mathrm{H}^{2}\left(M, \mathrm{G}_{\omega}\right) \rightarrow \mathrm{H}^{2}\left(M, \mathrm{G}_{d}\right)$ is exact.

14. In this $\mathrm{n}^{\circ}$, we assume $E=F$, and the principal symbol $\sigma(D)$ of $D$ does not vanish on any open set of $M$. We consider a smooth $\mathrm{G}$-valued function $g$ on $U$ to be a linear operator acting on $\mathrm{C}^{\infty}(U, E \otimes H)$. For differential operators $L$, $L_{1}, L_{2}: \mathrm{C}^{\infty}(U, E \otimes H) \rightarrow \mathrm{C}^{\infty}(U, E \otimes H)$, we set

$$
L^{g}=g^{-1} L g,\left[L_{1}, L_{2}\right]=L_{1} L_{2}+L_{2} L_{1} .
$$

Definition 26. Let $U$ be an open set of $M$ and $L: \mathrm{C}^{\infty}(U, E \otimes H) \rightarrow \mathrm{C}^{\infty}(U, E \otimes H)$ a differential operator of order $k-1, k=$ ord $D$, on $U$. Then we define a differential operator $D^{\mathrm{e}}(L): \mathrm{C}^{\infty}(U, E \otimes H) \rightarrow \mathrm{C}^{\infty}(U, E \otimes H)$ by

$$
D^{\mathrm{e}}(L)=\left(D \otimes 1_{H}+L\right)^{2}-D^{2} \otimes 1_{H}=L^{2}+\left[D \otimes 1_{H}, L\right] .
$$

$D^{\mathrm{e}}(L)$ was denoted by $\rho_{D}(L)$ in [6]. The following Lemma is also given in [6].

Lemma $9 . \quad D^{\mathrm{e}}$ has following properties

$\left(37\right.$, i) $\quad D^{\mathrm{e}}(c L)=c D^{\mathrm{e}}(L)+\left(c^{2}-c\right) L^{2}, c$ is a constant,

(37, ii) $\quad D^{\mathrm{e}}\left(L_{1}+L_{2}\right)=D^{\mathrm{e}}\left(L_{1}\right)+D^{\mathrm{e}}\left(L_{2}\right)+\left[L_{1}, L_{2}\right]$,

$(37, \text { ii })^{\prime} \quad D^{\mathbf{e}}\left(L_{1}-L_{2}\right)=D^{\mathrm{e}}\left(L_{1}\right)-D^{\mathbf{e}}\left(L_{2}\right)-\left[L_{1}-L_{2}, L_{2}\right]$,

(37, iii) $\quad D^{\mathrm{e}}\left(L^{g}\right)=D^{\mathrm{e}}(L)^{g}-\left[\rho_{D}(g), L^{g}\right]$,

(37, iv) $\quad D^{\mathrm{e}}\left(\rho_{D} g\right)=\rho_{D} 2(g)$. 
Proof. (37, i) and (37, ii) follow from Definition. (37, ii) follows from (37, i) and $(37$, ii). Since we have

$$
\begin{aligned}
D^{\mathrm{e}}\left(L^{g}\right) & =\left(D \otimes 1_{H}\right) L^{g}+L^{g}\left(D \otimes 1_{H}\right)+\left(L^{2}\right)^{g} \\
& =\left(\left(D \otimes 1_{H}\right) L^{g}+L^{g}\left(D \otimes 1_{H}\right)+L^{2}\right)^{2}+\left[D \otimes 1_{H}-\left(D \otimes 1_{H}\right)^{g}, L^{g}\right],
\end{aligned}
$$

we get (37, iii). (37, iv) foollows from Definition.

Corollary 1. We have

$$
D^{\mathrm{e}}\left(L^{g}+\rho_{D}(g)\right)=D^{\mathrm{e}}(L)^{g}+\rho_{D^{\mathrm{2}}(g)}
$$

Proof. By (37, ii) and (37, iv), we get

$$
D^{\mathrm{e}}\left(L^{g}+\rho_{D}(g)\right)=D^{\mathrm{e}}\left(L^{g}\right)+\rho_{D^{2}}(g)+\left[\rho_{D}(g), L^{g}\right] .
$$

Hence we have (38) by (37, iii).

Corollary 2. If $\rho_{D}(g)$ is $L_{2}-L_{1}{ }^{g}, D^{\mathrm{e}}\left(L_{2}\right)$ is equal to $D^{\mathrm{e}}\left(L_{1}\right)^{g}+\rho_{D^{2}}(g)$. Especially, if $D^{2}=0, D^{\mathrm{e}}\left(L_{2}\right)$ is equal to $D^{\mathrm{e}}\left(L_{1}\right)^{g}$.

Proof. Set $L_{2}=L_{1}{ }^{g}+\rho_{D}(g)$ and apply (38), we have Corollary.

Note. The reason of notational inhomogenity of $\rho_{D}$ and $D^{\mathbf{e}}$ was exposed in $n^{\circ} 1$. For functions, $D^{\mathrm{e}}(f)$ is defined by $\rho_{D}\left(\mathrm{e}^{f}\right)$ (cf. [8]). We also note if $f$ is a $g$-valued function, we say say $f$ to be a $c(D)$-class function if $f\left(D \otimes 1_{H}\right)=\left(D \otimes 1_{H}\right) f$ (cf [6], [8]).

The next Lemma generalizes gauge transformation

Lemma 10. Let $g$ be a smooth $\mathrm{G}-$ valued function on $U$ and $L: \mathrm{C}^{\infty}(U, E \otimes H)$ $\rightarrow \mathrm{C}^{\infty}(U, E \otimes H)$ a differential operator. We set

$$
g_{D}(L)=L^{g-1}+\rho_{D}\left(g^{-1}\right)=g\left(L-\rho_{D}(g)\right) g^{-1} .
$$

Then $g_{D}$ is a $\mathrm{G}$-action and we have

$$
D^{\mathrm{e}}\left(g_{D}(L)\right)=g_{D^{2}}\left(D^{\mathrm{e}}(L)\right) .
$$

Proof. Since $\mathrm{e}_{D}(L)=L$, to show $g_{D}$ to be a G-action, we need only to show $g_{D}\left(h_{D}(L)\right)=(g h)_{D}(L)$. But this follows from $g\left(h L h^{-1}-h \rho_{D}(h) h^{-1}\right) g^{-1}-g \rho_{D}(g) g^{-1}=(g h) L$ $(g h)^{-1}-g h \rho_{D}(h)(g h)^{-1}-g \rho_{D}(g) g^{-1}=(g h) L(g h)^{-1}-g h \rho_{D}(g h)(g h)^{-1}$. Since $g_{D}$ is a Gaction, (40) follows from (38).

We set $\mathscr{D}_{E \otimes H}^{k-1}$ the sheaf of germs of differential operators $L: \mathrm{C}^{\infty}(U, E \otimes H) \rightarrow$ $\mathrm{C}^{\infty}(U, E \otimes H)$ with the order at most $k-1$. By definition, $\mathscr{M}^{1}{ }_{D}$ is a subsheaf of $\mathscr{D}_{E \otimes H}^{k-1} . D^{\mathrm{e}}$ induces a sheaf map (also denoted by $D^{\mathrm{e}}$ ) on $\mathscr{D}_{E \otimes H}^{k-1}$. Then $\mathscr{A}^{1} D^{2}$ is a

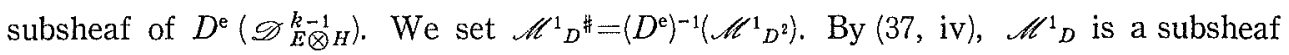
of $\mathscr{H}^{1} D^{\sharp}$. If $D^{2}=0, \mathscr{M}^{1} D^{\sharp}$ is the kernel sheaf oe $D^{\mathrm{e}}$. By the actions $g_{D}$ and $g_{D^{2}}{ }^{\prime}$ $\left(\mathscr{D}_{E \otimes H}^{k-1}\right)$. and $\left.D^{\mathrm{e}} \mathscr{D}_{E \otimes H}^{k-1}\right)$ are the $\mathrm{G}_{d^{-}}$-sheaves and $D^{\mathrm{e}}$ is an equivariant map by Lemma 10. Since $\mathscr{M}^{1}{ }^{2}$ is a $\mathrm{G}_{d^{-}}$-subsheaf of $D^{\mathrm{e}}\left(\mathscr{D}_{E \otimes H}^{k-1}\right), \mathscr{H}^{1} D^{\#}$ is a $\mathrm{G}_{d^{-}}$-subsheaf of 
$\mathscr{D}_{E \otimes H}^{k-1}$ and $\mathscr{M}^{1}{ }_{D}$ is a $\mathrm{G}_{d^{-}}$-subsheaf of $\mathscr{M}^{1}{ }^{\sharp}$.

Definition 27. The quotient sheaves of $\mathscr{H}^{1}{ }^{\sharp}, \mathscr{D}_{E \otimes H}^{k-1}$ and $D^{\mathrm{e}}\left(\mathscr{D}_{E \otimes H}^{k-1}\right)$ by $\mathrm{G}_{d^{-}}$ actions are denoted by $\mathscr{H}^{1} D^{\#}, \mathscr{D}_{E \otimes H}^{k-1}$, and $\mathscr{H}^{2}{ }_{D}$.

By Definitions, $D^{\mathrm{e}}$ induces the sheaf map $D^{\mathrm{e}}: \mathscr{D}_{E \otimes H}^{k-1} \rightarrow \mathscr{M}^{2}{ }_{D}$ and the following sequences are exact

$$
\begin{aligned}
& 0 \longrightarrow \mathscr{M}^{1} D^{\#-} \mathscr{D}_{E \otimes H}^{k-1} \stackrel{D^{\mathrm{e}}}{\longrightarrow} \mathscr{M}^{2}{ }^{2} \longrightarrow 0, \\
& 0 \longrightarrow \mathscr{M}^{1}{ } \longrightarrow \mathscr{M}^{1}{ }^{\#} \longrightarrow \mathscr{M}^{1} D^{\# \longrightarrow} \longrightarrow 0, \\
& 0 \longrightarrow \mathscr{H}^{1}{ }^{2} \longrightarrow D^{\mathrm{e}}\left(\mathscr{D}_{E \otimes H}^{k-1}\right) \longrightarrow \mathscr{H}^{2}{ }_{D} \longrightarrow 0 .
\end{aligned}
$$

Note. If $D^{2}=0, \mathscr{H}^{1} D^{2}$ is the 0 - sheaf. But $\mathscr{M}^{2}{ }_{D}$ may be different from $D^{\text {e }}$ $(\mathscr{D} \underset{E \otimes H}{k-1})$ since the $\mathrm{G}_{d}$-action on $D^{\mathrm{e}}\left(\mathscr{D}_{E \otimes H}^{k-1}\right)$ may not be trivial unless $n=1$. But the following sequence is exact if $D^{2}=0$.

$$
(2)_{D}^{\prime} \quad 0 \longrightarrow \mathscr{H}^{1} D^{\#} \longrightarrow \mathscr{D}_{E \otimes H}^{k-1} \stackrel{D^{\mathrm{e}}}{\longrightarrow} D^{\mathrm{e}}\left(\mathscr{D}_{E \otimes H}^{k-1}\right) \longrightarrow 0 .
$$

15. If $L \in H^{0}\left(M,\left(\mathscr{D}_{E \otimes H}^{k-1}\right), \bar{L}\right.$ has a resentative $\left\{L_{i}\right\} \in \mathrm{C}^{0} \mathfrak{U},\left(\mathscr{D}_{E \otimes H}^{k-1}\right)$ such that $L_{j}=g_{j i, D}\left(L_{i}\right),\left\{g_{j i}\right\} \in \mathrm{C}^{1}\left(\mathfrak{H}, \mathrm{G}_{d}\right)$. For $\xi=\left\{g_{i j}\right\}$, we set $\delta_{\xi, D}(L)_{i j}=L_{j}-g_{j i, D}\left(L_{i}\right)$.

Definition 28. Let $\xi=\left\{g_{i j}\right\}$ be in $\mathrm{C}^{1}\left(\mathfrak{H}, \mathrm{G}_{d}\right)$. Then we set

$\mathrm{H}_{d}^{0} M,\left(\mathscr{D}_{E \otimes H}^{k-1}\right)=\left\{L \in \mathrm{H}^{0}\left(M, \mathscr{D}_{E \otimes H}^{k-1}\right) \mid L=L_{i}, \quad \delta_{\xi, D} L=0\right.$ and $L_{i} t_{i j_{k}}=L_{i}$ for some $\left.\xi\right\}$

$D^{\mathrm{e}}$ induces a map from $\mathrm{H}^{0}\left(M, \mathscr{D}_{E \otimes H}^{k-1}\right)$ into $\mathrm{H}^{0}\left(M, \mathscr{M}^{2} D\right)$. We set

$$
\mathrm{H}^{0}{ }_{D}\left(M, \mathscr{H}^{2}{ }_{D}\right)=D^{\mathrm{e}}\left(\mathrm{H}_{d}^{0} d\left(M, \mathscr{D}_{E}^{k-1} \otimes H\right)\right)
$$

Theorem $\mathbb{1}_{D}$. There is a surjection $d R_{D}: \mathrm{H}^{0}{ }_{D}\left(M, \mathscr{M}^{2}{ }_{D}\right) \rightarrow \mathrm{H}^{1}\left(M, \mathscr{M}^{1} D\right)$.

Proof. If $\Theta \in H^{0}{ }_{D}\left(M, \mathscr{M}^{2}{ }_{D}\right), \Theta$ is represented by $\left\{D^{\mathrm{e}}\left(L_{i}\right\},\left\{L_{i}\right\} \in \mathrm{H}^{0}{ }_{d}\left(M, \mathscr{D}_{E \otimes H}^{k-1}\right)\right.$ Hence there exists $\left\{g_{i j}\right\} \in \mathrm{C}^{1} a\left(\mathfrak{U}, \mathrm{G}_{d}\right)$ such that to set $\omega_{i j}=L_{j}-L_{i} g_{j_{i}=\rho_{D}} g_{i j}$, we get $\omega_{j k}=\omega_{i k}+\omega_{i j}{ }^{g_{j k}}=0$. That is, $\left\{\omega_{i j}\right\}$ defines an element of $\mathrm{H}^{1}\left(M, \mathscr{H}^{\prime}{ }_{D}\right)$. If $\left\{D^{\mathrm{e}}\left(L_{i}{ }^{\prime}\right)\right\}$ is another representative of $\Theta$, we have $L_{i}{ }^{\prime}=h_{i, D}\left(L_{i}\right)$. Hence $\left\{\omega_{i j}{ }^{\prime}\right\}$ giues the same element of $\mathrm{H}^{1}\left(M, \mathscr{A}^{1}{ }_{D}\right)$ Therefore we can define the map $d R_{D}: \mathrm{H}^{0}{ }_{D}\left(M, \mathscr{H}^{2}{ }_{D}\right) \rightarrow \mathrm{H}^{1}$ $\left(M, \mathscr{M}^{1}{ }_{D}\right)$. If $\left\{g_{i j}\right\} \in \mathrm{C}^{1}{ }_{a}\left(\mathfrak{U}, \mathrm{G}_{d}\right)$ and $\left\{\omega_{i j}\right\}=\left\{\rho_{D}\left(g_{i j}\right)\right\}$ is in $\mathrm{Z}^{1}\left(\mathfrak{U}, \mathscr{H}^{1}{ }_{D}\right)$, by the same calculation as in the proof of Proposition 1, we get $\omega_{k i}{ }^{g_{i j}} g_{j k} g_{k i}=\omega_{k i}$. Hence we have

$$
\omega_{i j}=L_{j}-L_{i} g_{i j}, \quad L_{i}=\sum_{U_{k} \cap U_{i} \neq p} e_{k} \omega_{k i}
$$

Therefore $\left\{L_{i}\right\}$ represents an element of $\mathrm{H}_{d}^{0}\left(M, \mathscr{D}_{E \otimes H}^{k-1}\right)$ and we have $d R\left(\left\{L_{i}\right\}\right)=$ $\langle\omega\rangle$. Hence we have Theorem. 
Note 1. If $\xi=\left\{g_{i j}\right\}$ is a G-bundle, then above decomposition of $\omega_{i j}$ gives a connection of $D$ with respect to $\xi$ (cf. [1], [2], [5], [6]).

Note 2. If $D=\bar{\partial}$, denote $g^{1,0}$ and $g^{0,1}$ the sheaves of germs of smooth matrix valued $(1,0)$-and $(0,1)$-type forms, we have the following commutative diagram with exact lines and columns

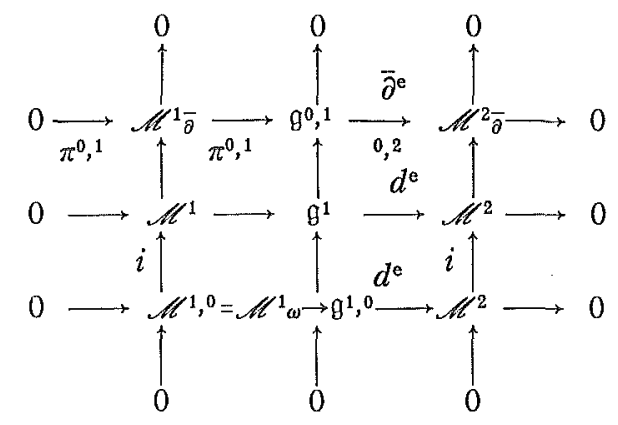

Hence $\langle\omega\rangle \in \mathrm{H}^{1}\left(M, \mathscr{M}^{1}\right)$ is in $i^{*}$-image if and only if there exists $\Theta \in Z^{0}{ }_{d}\left(M, \mathscr{M}^{2}\right)$ such that $\iota d R(\Theta)=\langle\omega\rangle$ and $\pi^{0},{ }^{2} \Theta=0$ (cf. [18]).

16. We assume $C_{c(D)}$ admits the following resolution (cf. [6])

$$
0 \longrightarrow \mathrm{C}_{c(D)} \stackrel{i}{\longrightarrow} \mathrm{C}_{d} \stackrel{d^{D}}{\longrightarrow} \mathrm{C}^{1, D} \stackrel{d^{D}}{\longrightarrow} \mathrm{C}^{2}, D \longrightarrow \cdots \cdots .
$$

We define the map $j: \mathscr{C}^{1}{ }_{D} \rightarrow \mathscr{M}^{1}{ }^{D}{ }^{D}$ by $j\left(\rho_{D}(g)\right)=\rho_{d} D(g)$. Then $j$ is abijection and therefore induces a bijection $j^{*}: \mathrm{H}^{1}\left(M, \mathscr{M}^{1}{ }_{D}\right) \rightarrow \mathrm{H}^{1}\left(M, \mathscr{M}^{1}{ }_{d} D\right)$. By the definition of $\mathscr{M}_{d}^{1} D, \Theta=\left\{\Theta_{i}\right\} \in Z_{d}^{0}\left(\mathfrak{U}, \mathscr{M}^{2}{ }_{d} D\right)$ is regarded to be $\Theta_{i} \in H^{0}\left(U_{i}, C^{2}, D \otimes \mathscr{Q}\right)$. Hence we may consider $\operatorname{tr}\left(\Theta_{\wedge \cdots \wedge} \stackrel{p}{ } \Theta\right.$ ) to be a $d^{D}$-closed $2 p$-form on $M$. The $d^{D}$-cohomology class of $\operatorname{tr}\left(\Theta_{\wedge \cdots}{ }^{D} \wedge \wedge\right)$ is determined by $\iota d R_{d}(\Theta) \in \mathrm{H}^{1}\left(M, \mathscr{M}^{1} d^{D}\right)$. Since $d R_{D}$ is onto, we can define the $p$-th $D$-Chern class $c^{p}{ }_{D}(\langle\omega\rangle)$ and the $p$-th $D$-Chern character $c h^{p}{ }_{D}(\langle\omega\rangle)$ by

$$
\begin{gathered}
c^{p}{ }_{D}(\langle\omega\rangle)=\text { the class of } c^{p}{ }_{D} \in \mathrm{H}^{2 p}\left(M, \mathrm{C} c_{(D)}\right) \\
c h^{p}{ }_{D}(\langle\omega\rangle)=\text { the class of }\left(\frac{\sqrt{-1}}{2 \pi}\right)^{p} \frac{1}{p !} \operatorname{tr}\left(\Theta_{\wedge \cdots \wedge}^{p} \Theta\right) \in \mathrm{H}^{2 p}\left(M, \mathrm{C}_{c(D)}\right) .
\end{gathered}
$$

Here, we set $\operatorname{det}(\mathbb{I}+(2 \pi / \sqrt{-1}))=\mathbb{I}+c^{1}{ }_{D} t+\cdots+c^{p}{ }_{D} t^{p}+\cdots+c^{n}{ }_{D} t^{n}$. Then, denoting $i_{D}: \mathbb{Z} \rightarrow \mathrm{C}_{c(D)}$ the inclusion, we have

$$
c^{p}{ }_{D}\left(\rho_{D} *(\xi)\right)=i_{D}^{*}\left(c^{p}(\xi)\right)
$$

$(26)^{\prime}{ }_{D}$

$$
\operatorname{ch}^{p}{ }_{D}\left(\rho_{D}{ }^{*}(\xi)\right)=i_{D}^{*}\left(\operatorname{ch}^{p}(\xi)\right) \text {. }
$$

In $[6]$, the right hand side of $(26)_{D}$ was called the $p$-th $c(D)$-characteristic class of $\xi \in \mathrm{H}^{\mathrm{1}}\left(M, \mathrm{G}_{d}\right)$. 
Example. If $M$ is a compact Kaehler manifold and $D=\bar{\partial}, c^{p} \bar{\partial}(\langle\omega\rangle)$ is a $(0,2 p)$ -type class. If $\langle\omega\rangle=\rho_{D}{ }^{*}(\xi), c^{p} \bar{\partial}(\langle\omega\rangle)$ is the $(0,2 p)$-type part of $c^{p}(\xi)$, the $p$-th Chern class of $\xi$.

By definition and $(26)_{D^{\prime}}$ we have

Proposition $3_{D}$. Let $\langle\omega\rangle$ be an element of $\mathrm{H}^{1}\left(M, \mathscr{M}^{1}{ }_{D}\right)$. Then $\langle\omega\rangle$ can not be in $\rho_{D}{ }^{*}$-image if $c^{p}{ }_{D}(\langle\omega\rangle)$ is not an integral class for some $p$.

\section{Bibliography}

[1] Andersson, S. I. : Vector bundle connections and lifting of partial differential operators, Differential Geometric Method in Mathematical physics, Clausthal 1980, Leot. Notes in Math., 905, 119-132, Berlin, 1982.

[2] Andersson, S. I.: Pseudodifferential operator and characteristic classes for nonabelian cohomology, Symposium on Differential Geometry, Peñiscola, 1982, Lect. Notes in Math., 1045, 1-10. Berlin, 1984.

[3] Asada, A. : Connection of flat vector bundles, J. Fac. Sci. Shinshu Univ. 2 (1967), 109-116.

[4] Asada, A. : Currents and residue exact sequences, J. Fac. Sci. Shinshu Univ., 3 (1968), 85-151.

[5] Asada, A.: Connection of differential operators, J. Fac. Sci. Shinshu Univ., 13 (1978), 87-102.

[6] Asada, A.: Flat connections of differential operators and odd dimensional characteristic classes, J. Fac. Sci. Shinshu Univ., 17 (1982), 1-30

[7] Asada, A.: Curvature forms with singularities and non-integral charateristic classes, Differential Geometric Method in Theoretical Physics, Clausthal, 1983, Lect. Notes in Math., 1139, 152-168, Berlin, 1985.

[8] Asada, A. Flat connections of differential operators and related characteristic classes, Differential Geometric Method in Theore tical Physics, Shumen, 1984.

[9.] Asada, A.: Non-abelian de Rham theories, Colloquim on Differential Geometry, Hajduszoboslo, 1984.

[10] Asada, A.: Non-abelian Poincaré lemma, Symposium on Differential Geometry, Peñiscola, 1985.

[11] Callan Jr., C. G.-Dashen, R.-Gross, D. J. : Toward a theory of the strong interaction, Physics Review D, 17 (1978), 2717-2763.

[12] Chern, S. S.: Characteristic classes of Hermitian manifolds, Ann. Math., 47 (1946), $85-121$.

[13] Dedecker, P.: Sur la cohomologienon abélienne, I, II, Canad. J. Math., 12 (1960), 231-251, 15 (1963), 84-93.

[14] Dedecker, P.-Luks, E. M.: Sur la non-fonctionalité du $\mathrm{H}^{2}$ en cohomologie non abélienne, C. R. Acad. Sci. Paris, 282 (1976), A 139-141.

[15] Gaveau, B.: Intégrales harmoniques non abéliennes, Bull. Sc. math., $2^{\mathrm{e}}$ série 106 (1982), 113-169. 
[16] Giraud, J.: Cohomologie non abélienne, Berlin, 1971.

[17] Grifone, J.: Connexions singuliéres et classe de Maslov, C. R. Acad. Sc. Paris 295 (1982), 139-142.

[18] Koszul, J. L. -Malgrange, B. : Sur certaines structure fibrés complexes, Arch. Math., 9 (1958), $102-109$.

[19] Lavendhomme, R.-Roissin, J. R.: Note on non-abelian cohomology, Applications of Sheaves, Durham, Lect. Notes in Math., 753, 534-541, Berlin, 1979.

[20] Mostow, M. A.: The field copy problem, Commun. Math. Phys., 78(1980), 137-150.

[21] Oniščik, A.: On the classification of fibre spaces, Soviet Math., Doklady 2 (1961), 1561-1564.

[22] Oniščik, A. : Connections with zero curvature and de Rham theorem, Soviet Math., Doklady 5 (1964), 1654-1657.

[23] Shaposnik, F. A.-Solomin, J. E. : Gauge field singularities and noninteger topological charge, J. Math. Phys., 20 (1979), 2110-2114.

[24] Schalafly, R.: A Chern number for gauge fields on R ${ }^{4}$, J. Math. Phys., 23 (1982), 1379-1384.

[25] Uhlenbeck, K. K.: The Chern classes of Sobolev connections, Commun. Math. Phys., 101 (1985), 449-457.

[26] Vassiliou, E.: Sur les connexions plates d'un fibré banachiques, C. R. Acad. Sci. Paris, 295 (1982), 353-356.

[27] Wen, X. G.-Witten, E.: Electric and magnetic charges in superstring models, Nucl. Phys., B 261 (1985), 651-677. 